Comparação dos projetos fatoriais completo e fracionado em um modelo de simulação de eventos discretos em um sistema de manufatura para os leiautes celular e celular virtual

\title{
Comparison of complete and fractured factor projects in a simulation model to discrete events of a manufacture system for virtual cellular and cellular layouts
}

Claudio Decker Junior ${ }^{1}$ - Universidade do Estado de Santa Catarina, Depto. Engenharia de Produção e Sistemas

Elisa Henning ${ }^{2}$ - Universidade do Estado de Santa Catarina, Depto. Matemática

João Carlos Espíndola Ferreira ${ }^{3}$ - Universidade Federal de Santa Catarina, Depto. Engenharia Mecânica

Bruna de Freitas Zappelino ${ }^{4}$ - Universidade do Estado de Santa Catarina, Depto. Engenharia de Produção e Sistemas

\section{RESUMO}

Este trabalho procura contribuir na escolha adequada do leiaute; tem por objetivo principal a aplicação de um Projeto Fatorial Fracionado e um Planejamento Fatorial Completo na simulação de um sistema de manufatura. São comparados o leiaute celular tradicional e o celular virtual, oito fatores de controle e a variável resposta é a utilização de recursos. A análise estatística realizada incluiu a Análise Exploratória de Dados, Análise de Variância, Teste t de Student e construção de Intervalos de Confiança para a Média. Efetuou-se a Análise de Sensibilidade para identificar os fatores com maior contribuição na variável resposta. Os resultados indicam que os dois tipos de projetos fatoriais chegam aos mesmos resultados no leiaute celular virtual, leiaute este, que apresenta utilização superior dos recursos em relação ao tradicional e os fatores mais influentes são o tempo de manutenção e de chegada dos lotes. Para o leiaute tradicional, os fatores que mais contribuem na utilização dos recursos são, além do programa de manutenção, a quantidade de lotes simulados e seu tamanho. Na análise de sensibilidade, o tempo de manutenção é o fator com maior influência na utilização dos recursos.

Palavras-chave: Projeto de experimentos. Fatorial completo. Fatorial fracionado. Simulação de sistemas. Leiaute. Células virtuais.

\section{ABSTRACT}

The definition of the best type of layout for a production process is a complex process, given the specificities and available resources. This paper aims to contribute in this context and its main objective is to compare the application of a Fractional Factorial Project and Full Factorial Planning in the simulation of a manufacturing system. Two types of layout are compared, traditional and virtual cellular, with eight control factors, and the response variable is the use of resources. Statistical analysis included the Exploratory Data Analysis, Analysis of Variance (ANOVA), Student's t-Test and Construction of Confidence Intervals for the Mean. In addition, sensitivity analysis was performed to identify the factors with the greatest contribution to the response variable. The results of the analyses indicate that the Fractional Factorial Project and Full Factorial Planning produce the same results for the virtual cellular layout. The virtual cellular layout presents higher use of the resources than the traditional ones and the most influential factors are the time of maintenance and arrival of the lots. For the traditional layout, the factors contributing most to resource utilization are, in addition to the maintenance program, the number of simulated batches and their size. In the analysis of sensitivity in all analyses, maintenance time is the factor with the greatest influence on resource utilization.

Keywords: Experimental design. Full factorial. Fractional factor. Simulation of systems. Layouts. Virtual cells.

1.Rua Paulo Malschitzki, 200, Zona Industrial Norte, Joinville, SC, CEP: 89.219-710, claudiodecker@gmail.com; 2. elisa.henning@gmail.com; 3. jc.ferreira@gmail.com; 4. zappelino.bruna@gmail.com

DECKER JR., C.; HENNING, E.; FERREIRA, J.C.E.; ZAPPELINO, B.F. Comparação dos projetos fatoriais completo e fracionado em um modelo de simulação de eventos discretos em um sistema de manufatura para os leiautes celular e celular virtual. GEPROS. Gestão da Produção, Operações e Sistemas, v. 15, n. 2, p. 23 - 57, 2020.

DOI: $10.15675 /$ gepros.v15i2.2422
Editor Responsável: Prof. Dr. Hermes Moretti Ribeiro da Silva 
Comparação dos projetos fatoriais completo e fracionado em um modelo de simulação de eventos discretos em um sistema de manufatura para os leiautes celular e celular virtual

\section{INTRODUÇÃO}

Determinar o melhor tipo de leiaute tem sido um problema clássico para a engenharia. Cada tipo de arranjo físico diferente possui características únicas que podem melhorar ou piorar o resultado da organização. A concorrência globalizada impõe uma elevada pressão nos sistemas de manufatura (EKREN; ORNEK, 2008). Nos últimos anos, o mercado consumidor exigiu um rápido aumento da variedade das famílias de produtos, além de uma redução no seu ciclo de vida. Tais mudanças obrigam as organizações a aumentar sua capacidade de resposta (ARKAT; GHAHVE, 2014).

Para aumentar esta capacidade de resposta, mudanças no leiaute podem se revelar essenciais. De acordo com Khilwani et al. (2011), o tipo de leiaute tem por objetivo aumentar o fluxo, a fim de reduzir os custos e aumentar a eficiência da produção. Para analisar os diferentes tipos de leiaute e seus efeitos na produção pode-se simular as diferentes situações. Segundo Freitas Filho (2008), a simulação de um modelo permite entender a dinâmica de um sistema, assim como analisar e prever o efeito de mudanças que nele se introduzam. Neste contexto, Kleijnen et al. (2005) afirmam que por meio da aplicação do planejamento de experimentos (DOE) os resultados da análise de uma simulação podem ser melhor trabalhados. Assim, é possível analisar a melhoria do desempenho no processo, evitando a técnica da tentativa e erro para buscar soluções (MONTEVECHI et al., 2007).

A modelagem de sistemas é importante para propor uma mudança de layout e analisar a sua performance em termos de produtividade e os efeitos produzidos por cada fator envolvido (YADAV; JAYSWAL, 2019). Nessa ótica, as técnicas de DOE são eficientes para o sucesso das organizações industriais (ANTONY, 2014). Os desenhos fracionados são uma boa escolha quando existem muitos fatores a serem considerados e deseja-se aproveitar a eficiência de um desenho fatorial, mas existem restrições devido aos custos e/ou condições experimentais (DZIAK et al., 2012). Essas restrições estão presentes no cotidiano das empresas, assim é fundamental investigar alternativas e mostrar suas possibilidades. Para Polenghi et al. (2018), o surgimento do conceito de Indústria 4.0 reforçou e aumentou ainda mais a importância da simulação de sistemas de manufatura.

Sendo assim, o objetivo deste artigo foi comparar a aplicação de um Projeto Fatorial Fracionado e um Planejamento Fatorial Completo na simulação de um sistema de manufatura. 
Comparação dos projetos fatoriais completo e fracionado em um modelo de simulação de eventos discretos em um sistema de manufatura para os leiautes celular e celular virtual

Foram comparados dois tipos de leiaute, celular virtual e celular tradicional, buscando também identificar para quais condições o leiaute celular virtual alcança melhores resultados para variável resposta utilização de recursos.

\section{REFERENCIAL TEÓRICO}

Em sistemas de manufatura, o leiaute estabelece a localização física dos recursos, que incluem máquinas e pessoas. Além disso, o leiaute define como os itens processados devem fluir durante as operações. A escolha adequada da configuração do leiaute no chão de fábrica é fundamental para viabilizar e aumentar a competitividade de uma empresa (KILWHANI et al., 2011).

Visando aumentar a competitividade surge o conceito do leiaute celular, no qual cada célula é projetada para atender a uma determinada família de peças, que se utiliza de máquinas e ferramental similares. Dentro dessa célula, as peças vão da matéria-prima ao produto acabado (GREENE; SADOWISKI, 1982). Dentre as vantagens do leiaute celular, Ekren e Ornek (2007) citam a redução no tempo de preparação (setup), redução do trabalho em processo (WIP) e o aumento no fluxo das peças. Porém é notório que o leiaute celular não é tão apropriado quando o sequenciamento da produção pode mudar muito. Drolet et al. (2008) citam que esse comportamento pode ser resultado de alta variabilidade das famílias de peças.

Para solucionar esse ponto desfavorável surge o conceito do leiaute celular virtual. Para Mclean et al. (1982) uma célula virtual é composta por máquinas de diferentes departamentos que são agrupadas logicamente durante um certo intervalo de tempo. Essas células funcionam como células de manufatura tradicionais, porém fisicamente as máquinas estão dispostas de forma aleatória no chão de fábrica. A Figura 1 apresenta um exemplo de leiaute celular virtual, no qual três células virtuais são temporariamente formadas para processar uma ordem de produção cada, as figuras geométricas representam os quatro tipos de máquinas que existem no chão de fábrica. 
Comparação dos projetos fatoriais completo e fracionado em um modelo de simulação de eventos discretos em um sistema de manufatura para os leiautes celular e celular virtual

Figura 1 - Exemplo de leiaute celular virtual

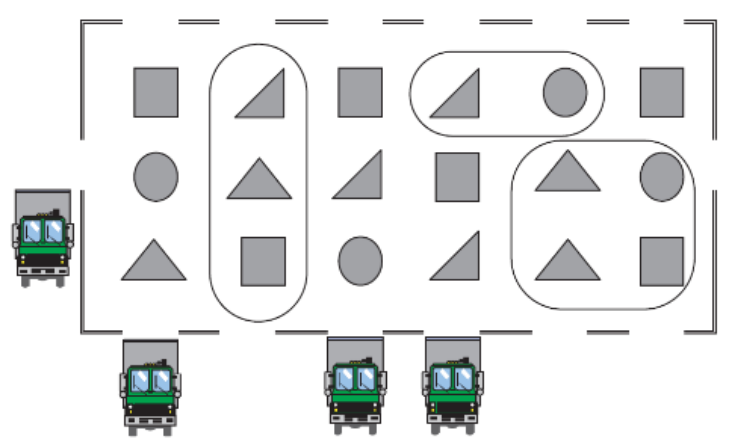

Fonte: Drolet et al. (2008).

Arkat e Ghahve (2014) ressaltam que o arranjo virtual temporário é frequentemente ajustado e definido de acordo com as características dos lotes das peças a serem fabricadas por meio do controle da produção e dos recursos disponíveis. Kilwhani et al. (2011) destacam em seu estudo uma proposta de maximização da similaridade entre os recursos e as peças, maximização do número de células e a minimização das sobreposições de células e do lead time. Essas quatro funções-objetivo foram desenvolvidas a partir de um modelo multiobjetivo e como resultado os benefícios das células virtuais se tornam mais evidentes com o acréscimo de recursos requeridos para processar determinado lote de peças.

Já Hamedi et al. (2012b) advertem que utilizar somente o tipo de recurso como critério para formação das células virtuais não é suficiente, principalmente porque algumas operações podem ser realizadas em mais de um tipo de recurso. Hamedi et al. (2012a) utilizam um modelo de programação não linear e os resultados alcançados para a capacidade dos sistemas foi superior para as células virtuais quando comparados com os das células tradicionais. Em outro estudo, Hamedi et al. (2012c) propõem um modelo matemático multiobjetivo por meio de programação baseada na abordagem de elementos-recurso. Por meio de um exemplo da literatura, Hamedi et al. (2012c) comprovam a partir do critério de capacidade de utilização dos recursos da célula que o desempenho das células virtuais foi superior.

Rezazadeh et al. (2009) propuseram um modelo de programação inteira mista para a formação das células virtuais envolvendo planejamento da produção, sistema de reconfiguração e os recursos necessários. O modelo proposto abrangia um algoritmo de otimização do tipo enxame de partículas (PSO). Em outra pesquisa, Jayachitra et al. (2010) 
Comparação dos projetos fatoriais completo e fracionado em um modelo de simulação de eventos discretos em um sistema de manufatura para os leiautes celular e celular virtual

apresentam um modelo estocástico dinâmico para projetar as células virtuais que minimiza o custo total do sistema de manufatura através da lógica Fuzzy.

Para Gomes (2010) e para Costa (2010), um problema experimental deve ser sustentado pelo projeto de experimentos e pela análise estatística dos dados. Para identificar se um ou mais fatores e suas interações produzem efeito na variável resposta aplica-se a Análise de Variância (ANOVA). Para complementar, é possível realizar a Análise de Sensibilidade a partir da ANOVA resultante do planejamento de experimentos. A análise de sensibilidade é o estudo da importância relativa de diferentes fatores de entrada na saída do modelo. Em outras palavras, se mais fatores produzem efeito significativo na variável resposta, é possível quantificar a importância de cada um e sinalizar os mais influentes (HAMBY, 1994; SALTELLI, 2002).

A integração da simulação de sistemas de manufatura com outras técnicas mostrou um crescimento nas últimas décadas (NEGAHBAN, 2014). O uso de técnicas de planejamento de experimentos aparece em aproximadamente 30\% dos artigos sobre o tema publicados em eventos e periódicos entre 1990 e 2016 (SOUSA JR et al., 2019).

\section{PROCEDIMENTOS METODOLÓGICOS}

Os fatores de controle e seus respectivos níveis estão representados resumidamente na Tabela 1.

Tabela 1 - Fatores de controle do experimento

\begin{tabular}{|c|c|c|c|c|}
\hline & Fator & Descrição & Nível & Valor \\
\hline \multirow{2}{*}{ F1 } & \multirow{2}{*}{ Features por peça } & \multirow{2}{*}{ Quantidade de features por peça } & Baixo & 1 a 3 features por peça \\
\hline & & & Alto & 7 a 10 features por peça \\
\hline \multirow{2}{*}{ F2 } & \multirow{2}{*}{ Features duplicadas } & \multirow{2}{*}{$\begin{array}{l}\text { Quantidade de duplicações de uma mesma } \\
\text { feature em cada peça }\end{array}$} & Baixo & 1 a 3 features \\
\hline & & & Alto & 7 a 10 features \\
\hline \multirow{2}{*}{ F3 } & \multirow{2}{*}{ Tamanho de lote } & \multirow{2}{*}{ Quantidade de peças em cada lote } & Baixo & 10 a 50 peças \\
\hline & & & Alto & 200 a 500 peças \\
\hline \multirow{2}{*}{ F4 } & \multirow{2}{*}{ Tipo de demanda } & \multirow{2}{*}{$\begin{array}{l}\text { O tipo de demanda ao qual o sistema será } \\
\text { submetido }\end{array}$} & Baixo & Aleatória \\
\hline & & & Alto & Controlada \\
\hline \multirow{2}{*}{ F5 } & \multirow{2}{*}{ Tipo de plano } & \multirow{2}{*}{$\begin{array}{l}\text { Plano de processos utilizado na simulação } \\
\text { com ou sem alternativas }\end{array}$} & Baixo & Plano SEM alternativas \\
\hline & & & Alto & Plano COM alternativas \\
\hline \multirow{2}{*}{ F6 } & \multirow{2}{*}{ Lotes simulados } & \multirow{2}{*}{$\begin{array}{l}\text { Quantidade total de lotes simulados em } \\
\text { cada replicação }\end{array}$} & Baixo & 50 lotes \\
\hline & & & Alto & 200 lotes \\
\hline \multirow{2}{*}{ F7 } & \multirow{2}{*}{ Tempo de chegada } & \multirow{2}{*}{ Tempo para a chegada do próximo lote } & Baixo & $5 \%$ a $10 \%$ do tempo de fabricação \\
\hline & & & Alto & $20 \%$ a $30 \%$ do tempo de fabricação \\
\hline \multirow{2}{*}{ F8 } & \multirow{2}{*}{ Tempo de manutenção } & \multirow{2}{*}{ Tempo de manutenção dos equipamentos } & Baixo & Modelo SEM manutenção \\
\hline & & & Alto & Modelo COM manutenção \\
\hline
\end{tabular}


Comparação dos projetos fatoriais completo e fracionado em um modelo de simulação de eventos discretos em um sistema de manufatura para os leiautes celular e celular virtual

Fonte: Decker Junior et al. (2015).

A variável resposta deste estudo será a utilização de recursos (U), que é calculado pela razão entre o período em que o recurso foi utilizado $(\Delta t u)$ em um determinado intervalo de tempo disponível $(\Delta t)$, medido percentualmente $(\%)$. Para ambos os leiautes são considerados planos de processo com e sem alternativas. A fim de reduzir os erros inerentes à variabilidade do sistema, 10 simulações foram realizadas para cada combinação de fatores, ou seja, cada experiência foi replicada 10 vezes (DECKER JUNIOR et al., 2015).

Para o projeto fatorial completo, todas as combinações possíveis foram experimentadas. Considerando oito fatores de controle (Tabela 1), com dois níveis em cada fator (baixo e alto), foram realizados $2^{8}=256$ experimentos. Para cada experimento foram realizadas 10 simulações, 2.560 experimentos, e cada um desses experimentos foi aplicado a dois leiautes diferentes (leiautes celulares virtuais e tradicionais), totalizando 5.120 experimentos.

Para o projeto fatorial fracionado $2^{(k-p)}$, a ordem estabelecida foi 1 , ou seja, $p=1$. Com isso houve uma redução nos experimentos realizados a metade, pois $2^{(8-1)}=128$ combinações. Também se realizaram 10 simulações para cada experimento, originando 1.280 experimentos totais para os dois tipos de leiaute. Montgomery (2009) sugere que ao menos duas replicações sejam feitas e, com isso, determina-se a soma dos quadrados (SS) decorrente do erro. Visando reduzir erros inerentes à variabilidade do sistema, foram realizadas 10 simulações para cada combinação de fatores, ou seja, cada experimento foi replicado 10 vezes, tendo em vista a capacidade do hardware e o tempo disponível para a simulação.

Para determinar quais seriam as combinações utilizadas aplicou-se a resolução do tipo III, pois o interesse estava no comportamento dos fatores principais.

A resolução de um experimento é uma propriedade dos experimentos fatoriais fracionados relacionada com a ordem dos efeitos que estão confundidos. Em experimentos fatoriais fracionados, um ou mais efeitos são confundidos, ou seja, não podem ser estimados separadamente. Num planejamento de resolução III nenhum efeito principal está associado com qualquer outro efeito principal, porém efeitos principais estão associados com interações de dois fatores (MONTGOMERY, 2009). 
Comparação dos projetos fatoriais completo e fracionado em um modelo de simulação de eventos discretos em um sistema de manufatura para os leiautes celular e celular virtual

\subsection{Estágios da simulação}

Seguindo a proposta de Decker Junior et al. (2015), a construção do modelo foi feita em duas etapas. Na primeira são geradas as ordens de produção dos lotes de peças. Nesse estágio são realizadas as seguintes atividades: geração das características das peças, definição da quantidade de recursos duplicados, definição do tamanho do lote, definição do tipo de demanda, geração da sequência de usinagem e cálculo dos tempos de usinagem. Esses dados são armazenados em uma planilha usada como dado de entrada no software de simulação Arena®.

Na segunda etapa, a simulação é realizada e os resultados também são armazenados em uma planilha que será usada para análise estatística. O diagrama do fluxo na Figura 2 ilustra essas duas etapas.

Figura 2 - Estrutura do modelo de simulação
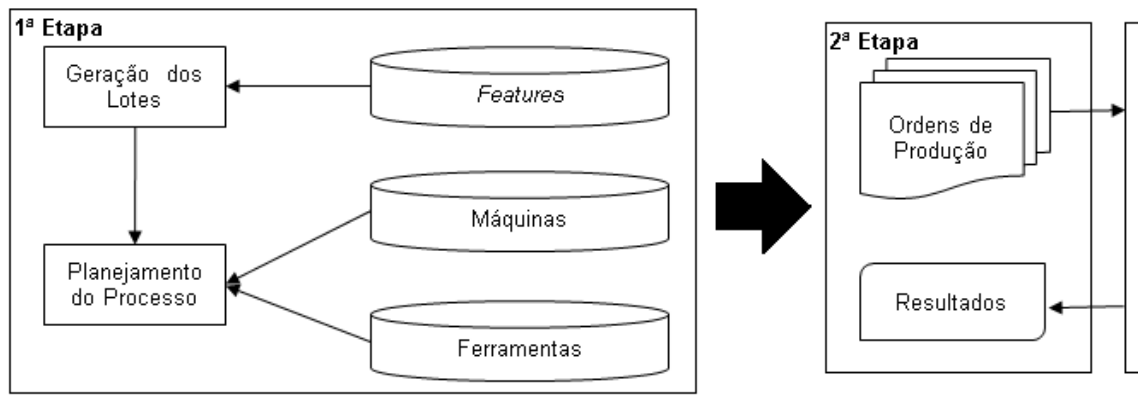

Modelo Sistema Manufatura

Leiaute Celular Tradicional: Plano COM ou SEM alternativa

Leiaute Celular Virtual: Plano COM ou SEM alternativa

Fonte: Decker Junior et al. (2015).

Os modelos de cada leiaute contêm o mesmo número de máquinas, que podem executar determinadas operações. São duas furadeiras, duas fresadoras convencionais, duas fresadoras $\mathrm{CNC}$, dois tornos convencionais e dois tornos $\mathrm{CNC}$, totalizando 10 máquinas. Além disso, existem oito operadores e três movimentadores. No total, cada modelo tem 21 recursos.

Nas Figuras 3 e 4, uma representação da animação dos leiautes utilizados na simulação. 
Comparação dos projetos fatoriais completo e fracionado em um modelo de simulação de eventos discretos em um sistema de manufatura para os leiautes celular e celular virtual

Figura 3 - Animação do leiaute celular virtual

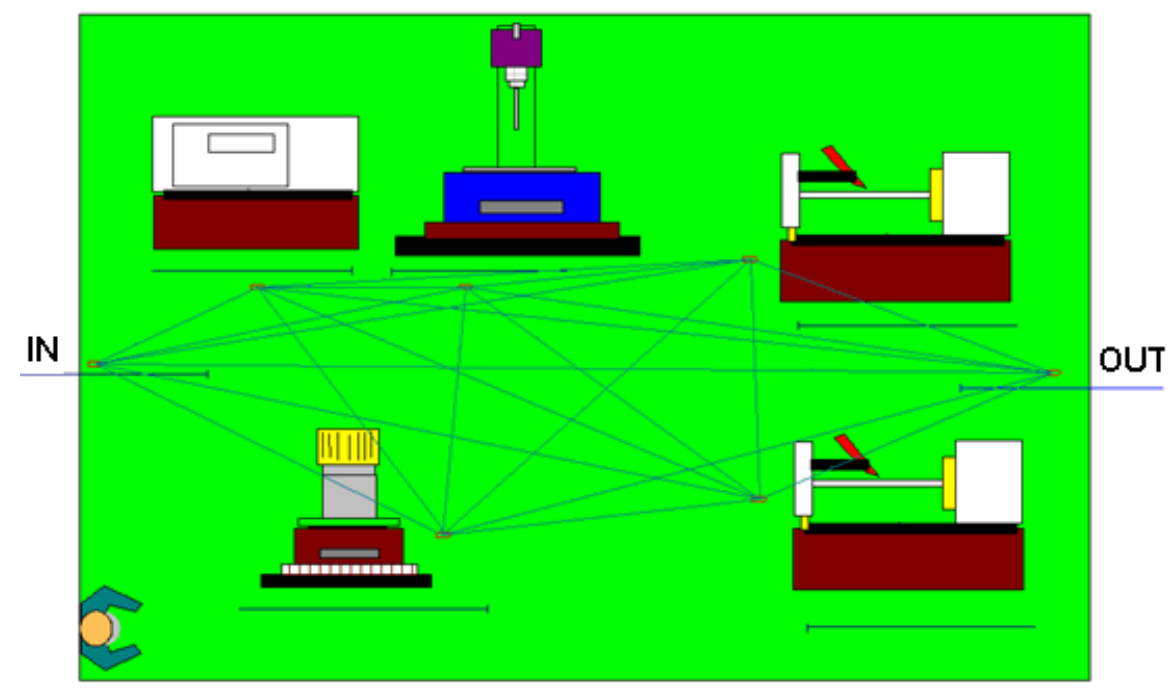

Fonte: Decker Junior (2015).

Figura 4 - Animação do leiaute celular tradicional

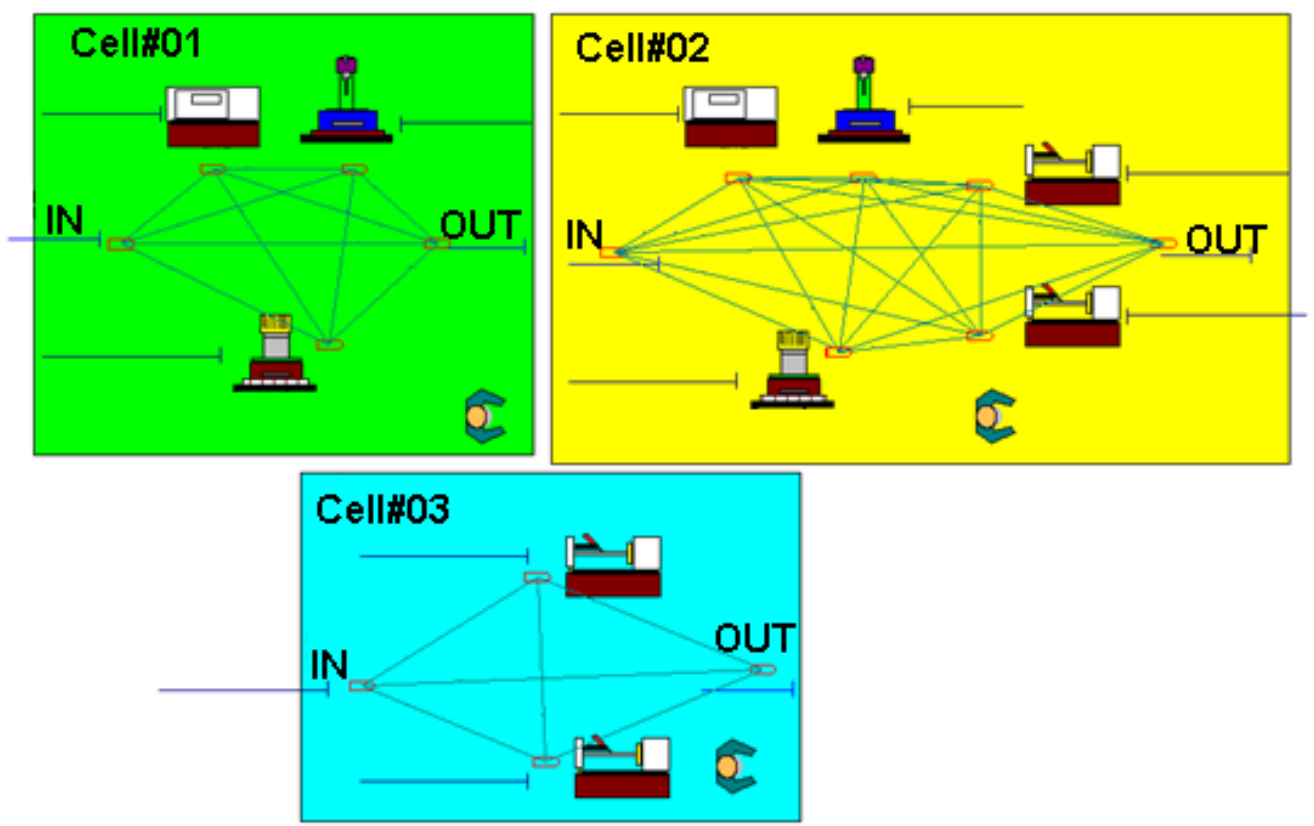

Fonte: Decker Junior (2015).

Os movimentadores são responsáveis pelo transporte dos lotes de peças da entrada de cada sistema de fabricação para cada máquina e, eventualmente, pela sua entrega à área de 
Comparação dos projetos fatoriais completo e fracionado em um modelo de simulação de eventos discretos em um sistema de manufatura para os leiautes celular e celular virtual

expedição. A Tabela 2 mostra os tipos diferentes de máquinas e as operações que cada máquina pode executar.

Tabela 2 - Tipos de máquinas e suas operações

\begin{tabular}{c|c|c|c} 
Máquina & Operação & $\begin{array}{c}\text { Plano de processo } \\
\text { SEM alternativa }\end{array}$ & Plano de processo COM alternativa \\
\hline $\mathbf{1}$ & Furadeira & Furo & Furo \\
\hline $\mathbf{2}$ & Fresadora & Rebaixo, canal & Furo, rebaixo, canal e cavidade \\
\hline $\mathbf{3}$ & Fresadora CNC & Cavidade & Furo, rebaixo, canal e cavidade \\
\hline $\mathbf{4}$ & Torno & Recartilhar e sangrar & Tornear, facear, sangrar e recartilhar \\
\hline $\mathbf{5}$ & Torno CNC & Tornear e facear & Tornear, facear, sangrar e recartilhar
\end{tabular}

Fonte: Decker Junior et al. (2015).

As células foram criadas de acordo com o tipo de peça a ser fabricada: peças cilíndricas, prismáticas ou combinadas (com partes cilíndricas e prismáticas) Um dos fatores de controle é o tipo de demanda, que pode ser aleatória ou controlada. Para demanda controlada, a formação das peças é a seguinte: $20 \%$ de peças cilíndricas, $30 \%$ de peças prismáticas e $50 \%$ de peças combinadas. Foram estudados três tipos de células diferentes: a primeira célula fabrica apenas peças prismáticas, consistindo de apenas uma furadeira, uma fresadora convencional e uma fresadora CNC.

A segunda célula foi estabelecida para fazer peças que têm características cilíndricas e prismáticas. Esta célula será composta por uma furadeira, uma fresadora $\mathrm{CNC}$, uma fresadora convencional, um torno convencional e um torno CNC. A terceira célula foi projetada para fazer peças de formas cilíndricas, apenas, e tem um torno convencional e um torno CNC. Cada célula tem um movimentador que é responsável pela coleta da matéria-prima no início do processo e transporte do produto acabado para a saída de cada sistema. Este movimentador é dedicado à célula, não interage nem muda de célula.

No leiaute das células virtuais, com relação aos movimentadores, eles têm total liberdade para andar no chão de fábrica, desde a entrada da matéria-prima até a saída do lote de produtos acabados. Um dos fatores de controle utilizados nesta pesquisa foi o tipo de plano de processo. No caso do uso de um plano de processo com alternativas, uma segunda opção de máquina foi definida para executar a operação.

Para cada modelo foi necessário estabelecer alguns parâmetros relativos aos tempos de transporte. Para o leiaute celular tradicional foi utilizada uma distribuição uniforme de 3 a 6 
Comparação dos projetos fatoriais completo e fracionado em um modelo de simulação de eventos discretos em um sistema de manufatura para os leiautes celular e celular virtual

minutos para entrar na célula, e também uma distribuição uniforme de 3 a 6 minutos para iniciar a operação em cada recurso. Além dos tempos de transporte mencionados, foram especificadas as distâncias entre cada recurso, bem como a velocidade com que os carregadores executavam o transporte (DECKER JUNIOR et al., 2015).

Para o leiaute de célula virtual foi estabelecida uma distribuição uniforme com um valor de 30 a 60 minutos para o transporte entre departamentos e uma distribuição uniforme de 3 a 6 minutos para iniciar o processamento em lote no recurso selecionado. Em geral, a distância entre os equipamentos era de 5 metros, desde a entrada do sistema de fabricação até o equipamento era igual a 10 metros e a distância entre o equipamento e o setor de expedição (ou seja, saída) era de 20 metros. A velocidade com que o carregador transporta cada lote foi ajustada em $5 \mathrm{~m} / \mathrm{min}$ para todos os modelos (DECKER JUNIOR et al., 2015). Com relação aos tempos de setup, foram adotados os mesmos valores para ambos os modelos, os quais foram obtidos de Hamedi et al. (2012a): (a) furadeira e torno = 20 minutos; (b) fresadora, fresadora $\mathrm{CNC}$ e torno $\mathrm{CNC}=35$ minutos.

Os valores atribuídos ao tempo de troca da ferramenta foram estabelecidos de acordo com estudos relatados na literatura e dados dos catálogos de fabricantes. O tempo entre as mudanças de ferramenta de corte nas furadeiras, fresadoras CNC e fresadoras foi estabelecido como uma distribuição de Poisson com valor médio de $60 \mathrm{~min}$, e para o tempo de troca de ferramenta foi utilizada uma distribuição uniforme de 5-10 minutos. O intervalo entre as mudanças na ferramenta de corte para tornos $\mathrm{CNC}$ e tornos convencionais tem uma distribuição uniforme de 5 a 10 minutos, enquanto que uma distribuição de Erlang com média $\mu=2$ foi considerada para o tempo de troca de ferramenta (AL-AOMAR; AL-OKAILY, 2006).

Os tempos de manutenção estabelecidos segundo Wang et al. (2011) para o intervalo entre falhas foi uma distribuição de Weibull $\operatorname{com} \delta=17.151$ e $\alpha=71,4$ minutos (parâmetros de forma e escala) para as furadeiras, fresadoras e fresadoras $\mathrm{CNC}$, com tempo de reparo constante de 96 min. Já para os tornos e tornos CNC, segundo Devise e Pierreval (2000), o valor do intervalo entre falhas foi de 38.400 minutos, com reparo de 240 minutos.

As etapas e respectivas distribuições de probabilidade estão sumarizadas na Tabela 3. 
Comparação dos projetos fatoriais completo e fracionado em um modelo de simulação de eventos discretos em um sistema de manufatura para os leiautes celular e celular virtual

Tabela - Distribuições de probabilidade

\begin{tabular}{|c|c|c|}
\hline Variável & Distribuição & Parâmetros \\
\hline Celular tradicional: tempo de transporte (minutos) & Uniforme & $\mathrm{U}(\alpha=3, \beta=6)$ \\
\hline Celular virtual: tempo de transporte (minutos) & Uniforme & $\mathrm{U}(\alpha=30, \beta=60)$ \\
\hline $\begin{array}{c}\text { Tempo entre as mudanças de ferramenta de corte nas } \\
\text { furadeiras e fresadoras }\end{array}$ & Poisson & $\lambda($ média $)=60$ minutos \\
\hline $\begin{array}{l}\text { Tempo entre as mudanças na ferramenta de corte para os } \\
\text { tornos }\end{array}$ & Uniforme & $\mathrm{U}(\alpha=5, \beta=10)$ \\
\hline Tempo de troca de ferramenta & Erlang & $\mu=2$ \\
\hline Tempos de manutenção & Weibull & $\begin{array}{l}\delta=17151 \text { e } \alpha=71,4 \\
\text { minutos }\end{array}$ \\
\hline
\end{tabular}

Fonte: Os autores.

\subsection{Geração das ordens de produção}

A Figura 5 mostra um fluxograma com o programa que gera as ordens de produção. Considerando que os lotes de peças a serem fabricadas foram definidos na etapa anterior, é necessário que o modelo possa reproduzir as características desejadas. O cálculo do tempo de usinagem é realizado considerando-se o tipo de plano selecionado. Depois que os tempos são definidos, um movimentador é solicitado. O movimentador também é solicitado: (a) quando um lote precisa entrar no sistema; (b) para mover o lote para a próxima máquina; ou (c) para transportar o lote para a área de expedição (DECKER JUNIOR et al., 2015).

Figura 5 - Fluxograma para geração das ordens de produção

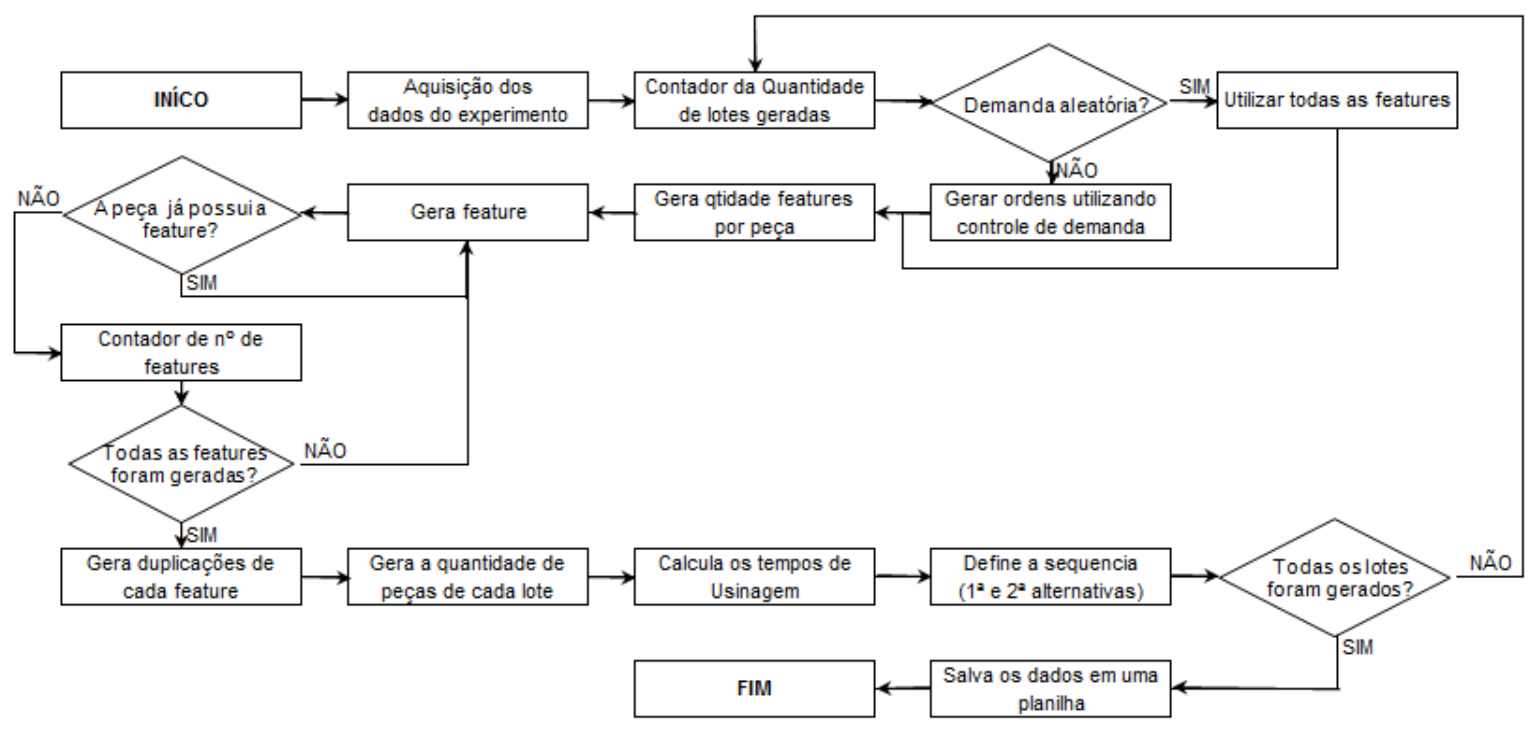

Fonte: Decker Junior et al. (2015). 
Comparação dos projetos fatoriais completo e fracionado em um modelo de simulação de eventos discretos em um sistema de manufatura para os leiautes celular e celular virtual

O lote sai da máquina selecionada somente quando todas as peças do lote são usinadas. No caso de planos de processo com alternativas, verifica-se a possibilidade de utilizar a segunda opção de máquina na próxima operação. Isso é feito comparando-se o estoque em processo no recurso selecionado para a próxima operação (primeira opção) com o recurso atual (segunda opção de máquina). Se a segunda opção de máquina selecionada para a próxima operação for igual à máquina atual, o trabalho em andamento é avaliado para evitar uma mudança de máquina, o que reduz significativamente o número de setups (DECKER JUNIOR et al., 2015). Se não houver mais operações, o lote sai do sistema, mas antes disso seus dados estatísticos são coletados para o cálculo da utilização dos recursos.

\subsection{Modelos de simulação}

A Figura 6 ilustra o modelo geral elaborado no software Arena, a partir dele foram extraídos os dados necessários para o cálculo das variáveis.

Figura 6 - Modelo leiaute celular virtual elaborado no Arena

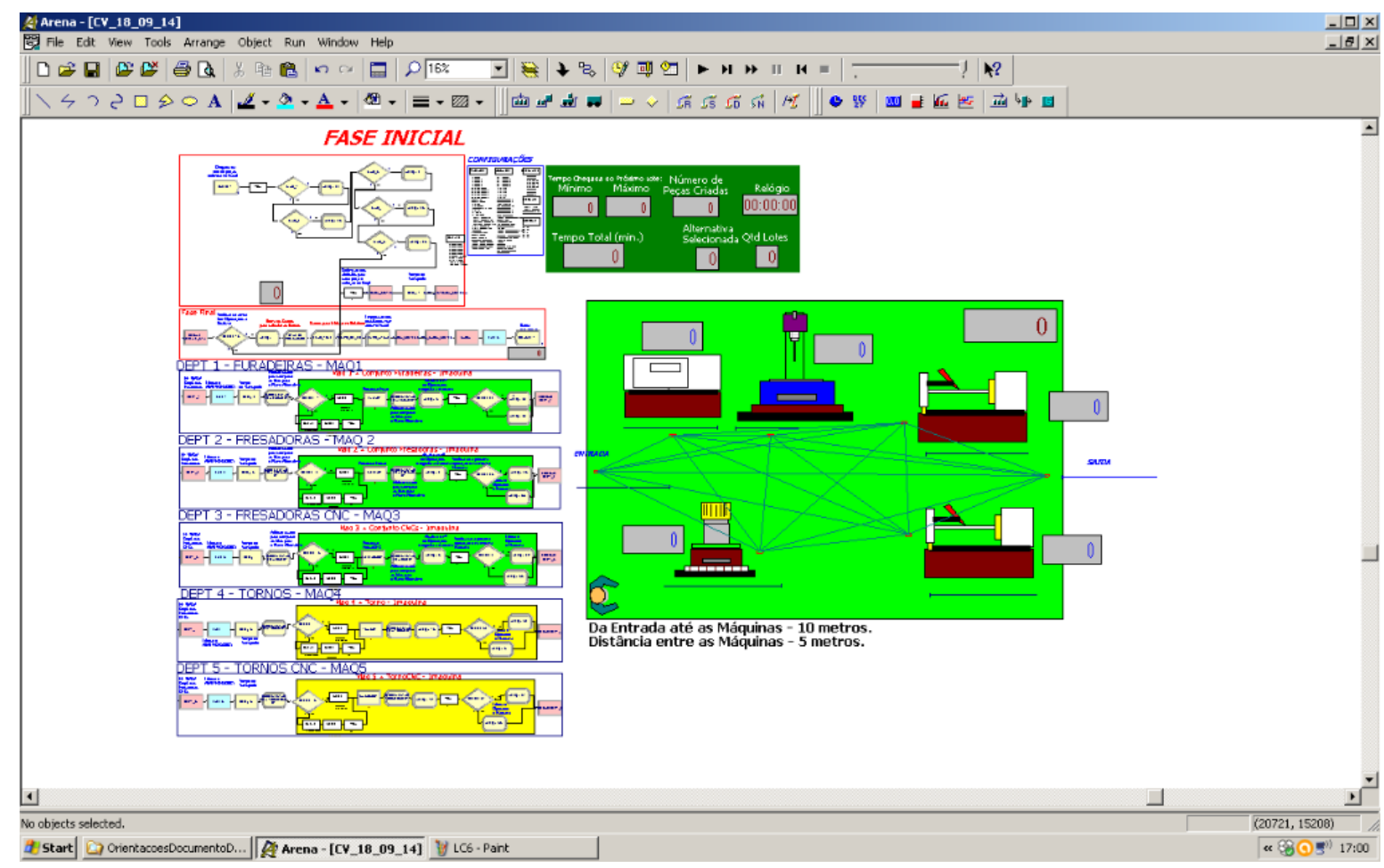

Fonte: Decker Junior (2015). 
Comparação dos projetos fatoriais completo e fracionado em um modelo de simulação de eventos discretos em um sistema de manufatura para os leiautes celular e celular virtual

A partir do modelo geral as figuras que seguem ilustram o detalhamento de cada parte do modelo. A Figura 7 apresenta a fase inicial do modelo celular virtual.

Figura 7 - Fase inicial do leiaute celular virtual elaborado no Arena

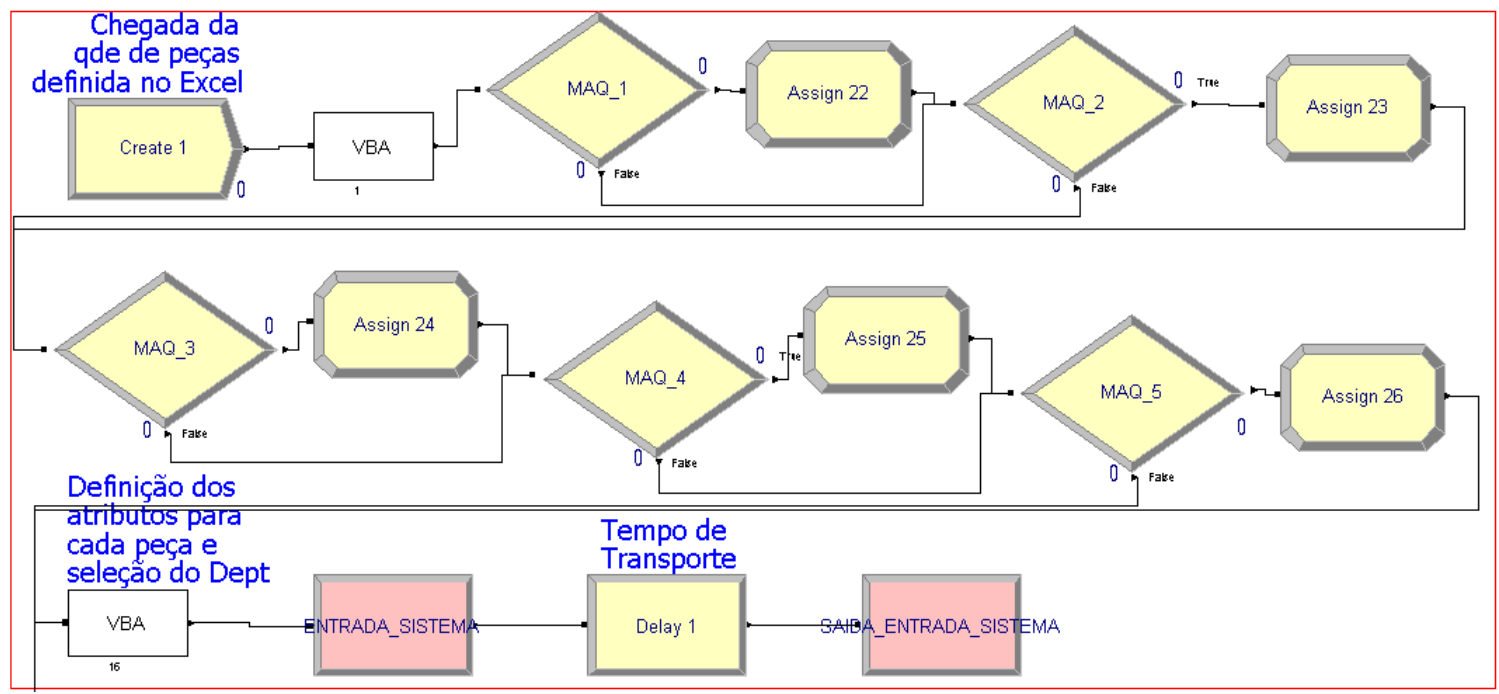

Fonte: Decker Junior (2015).

A Figura 8 ilustra a lógica de um dos departamentos do leiaute celular virtual.

Figura 8 - Exemplo de um departamento do leiaute celular virtual elaborado no Arena

\section{DEPT 3 - FRESADORAS CNC - MAQ3}

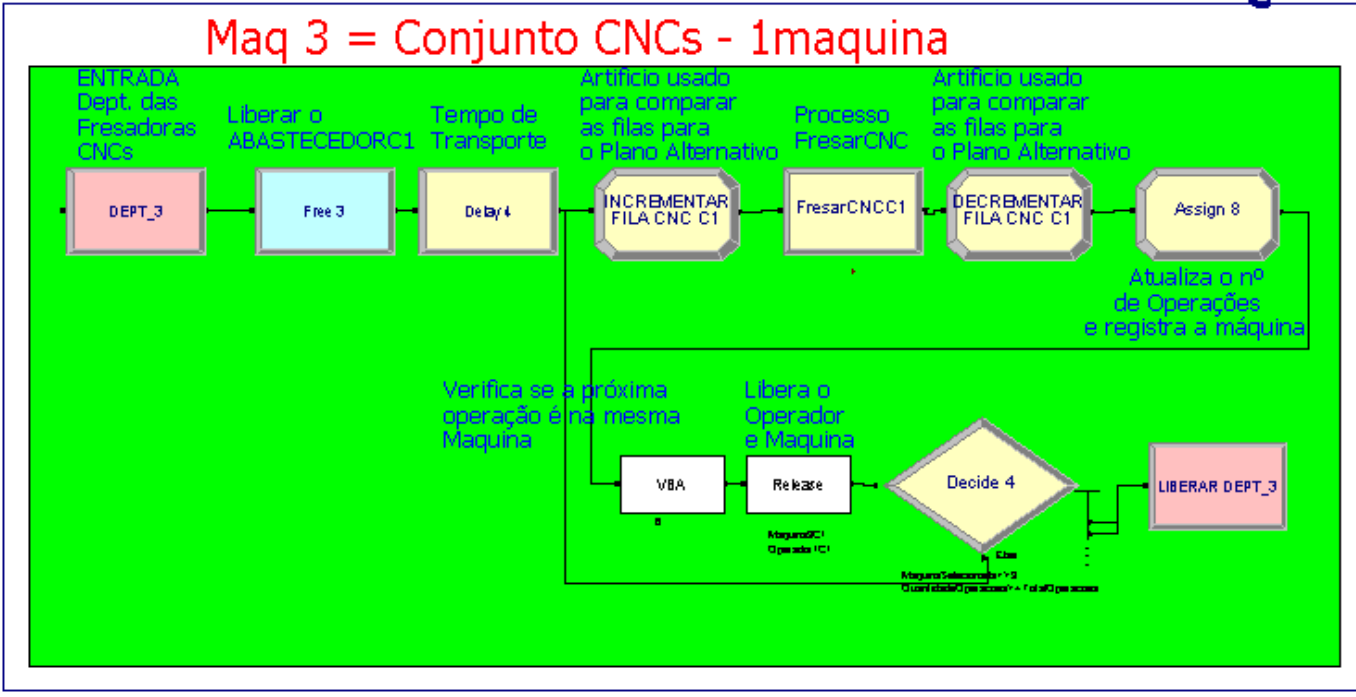

Fonte: Decker Junior (2015). 
Comparação dos projetos fatoriais completo e fracionado em um modelo de simulação de eventos discretos em um sistema de manufatura para os leiautes celular e celular virtual

Por fim, a Figura 9 ilustra a fase final do modelo do leiaute celular virtual. Sempre que um lote deixa o departamento, ele retorna à fase final, e nessa fase verifica-se inicialmente se todas as operações foram executadas. Se alguma operação ainda não foi executada, o movimentador leva o lote até o departamento. Caso contrário, se todas as operações foram executadas, o lote de peças é submetido ao cálculo de algumas das variáveis-resposta e então é finalizada a simulação daquele lote.

Figura 9 - Fase final do leiaute celular virtual elaborado no Arena

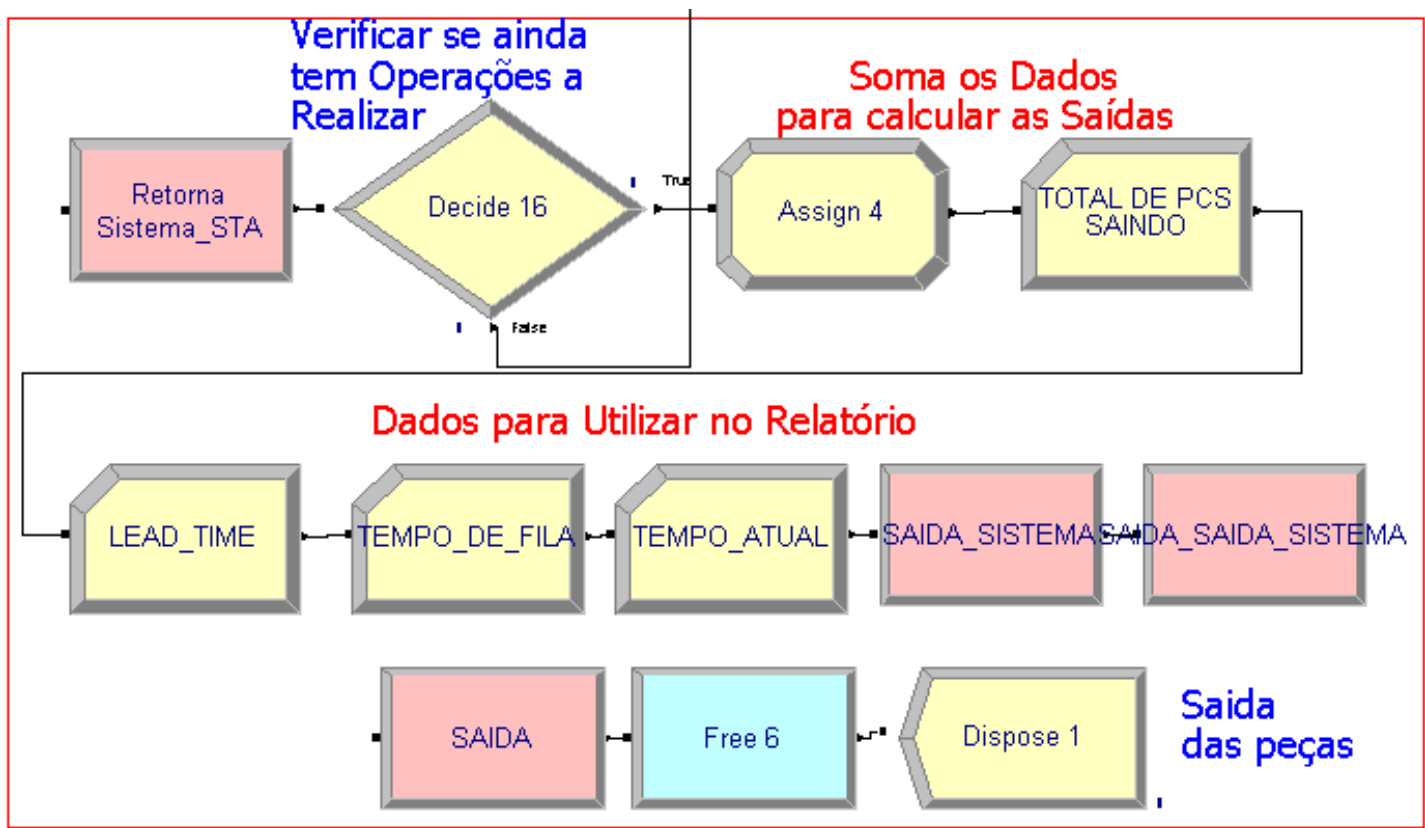

Fonte: Decker Junior (2015).

A Figura 10 ilustra a tela do modelo desenvolvido no Arena para simular o leiaute celular tradicional. 
Comparação dos projetos fatoriais completo e fracionado em um modelo de simulação de eventos discretos em um sistema de manufatura para os leiautes celular e celular virtual

Figura 10 - Modelo do leiaute celular tradicional elaborado no Arena

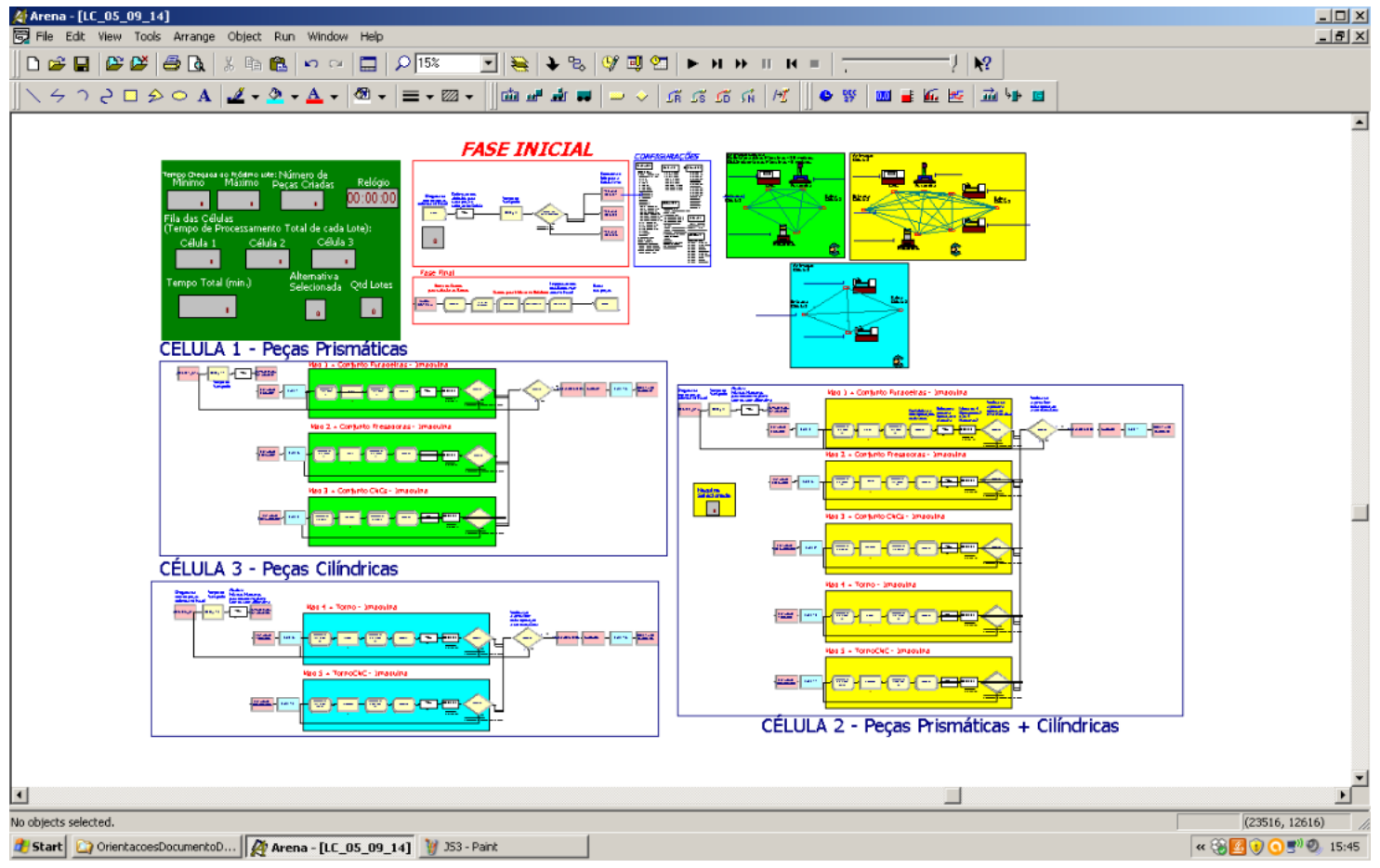

Fonte: Decker Junior (2015).

A Figura 11 ilustra a fase inicial do modelo celular tradicional. Nesta fase são definidos os atributos dos lotes de peças no programa em Visual Basic mediante o bloco "VBA 1", também responsável por direcionar o lote para a célula específica. Ou seja, o programa auxiliar em Visual Basic lê quais as máquinas que cada lote de peças precisa e então direciona o lote para a célula correta.

Figura 11 - Fase inicial do leiaute celular tradicional elaborado no Arena

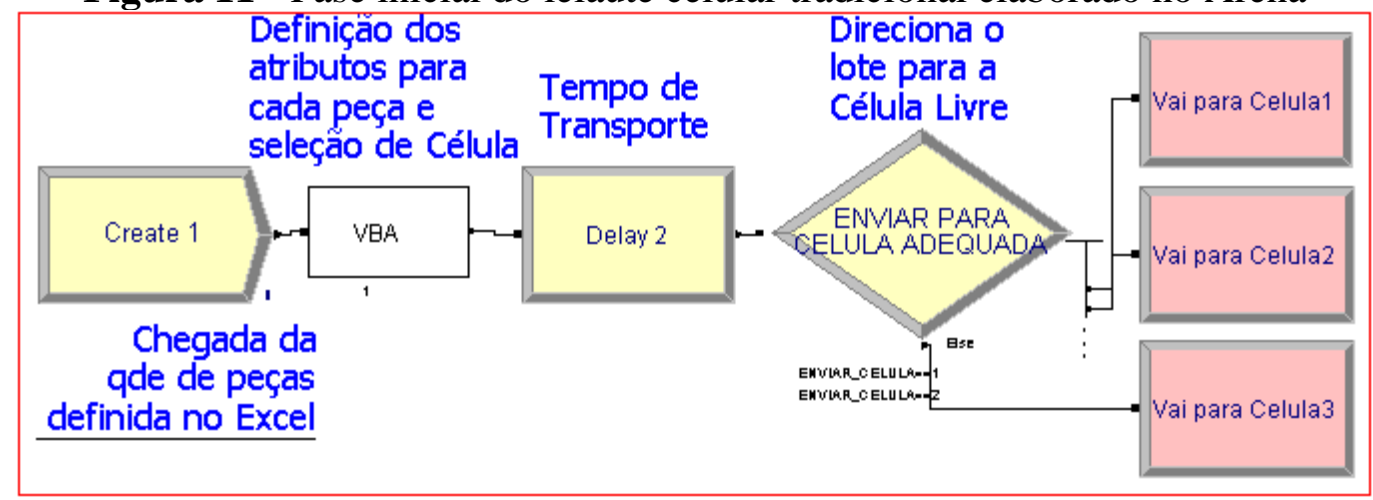

Fonte: Decker Junior (2015). 
Comparação dos projetos fatoriais completo e fracionado em um modelo de simulação de eventos discretos em um sistema de manufatura para os leiautes celular e celular virtual

As Figuras 12, 13 e 14 ilustram as três células. Analisando a célula 1 verifica-se a seguinte lógica: após o lote de peças chegar à célula adequada, o programa auxiliar verifica para qual máquina o lote deve ser enviado. Dessa forma, solicita-se o movimentador da célula e o mesmo leva o lote até a referida máquina, para que as operações sejam executadas.

Da mesma forma, como no leiaute celular virtual, a lógica das operações é a mesma. O lote é deslocado até a máquina e o operador executa as operações necessárias. Mediante o programa auxiliar são atualizados alguns dados, como a quantidade de operações já executadas, para qual máquina o lote irá posteriormente, etc.

Figura 12 - Célula 1 do leiaute celular tradicional elaborado no Arena

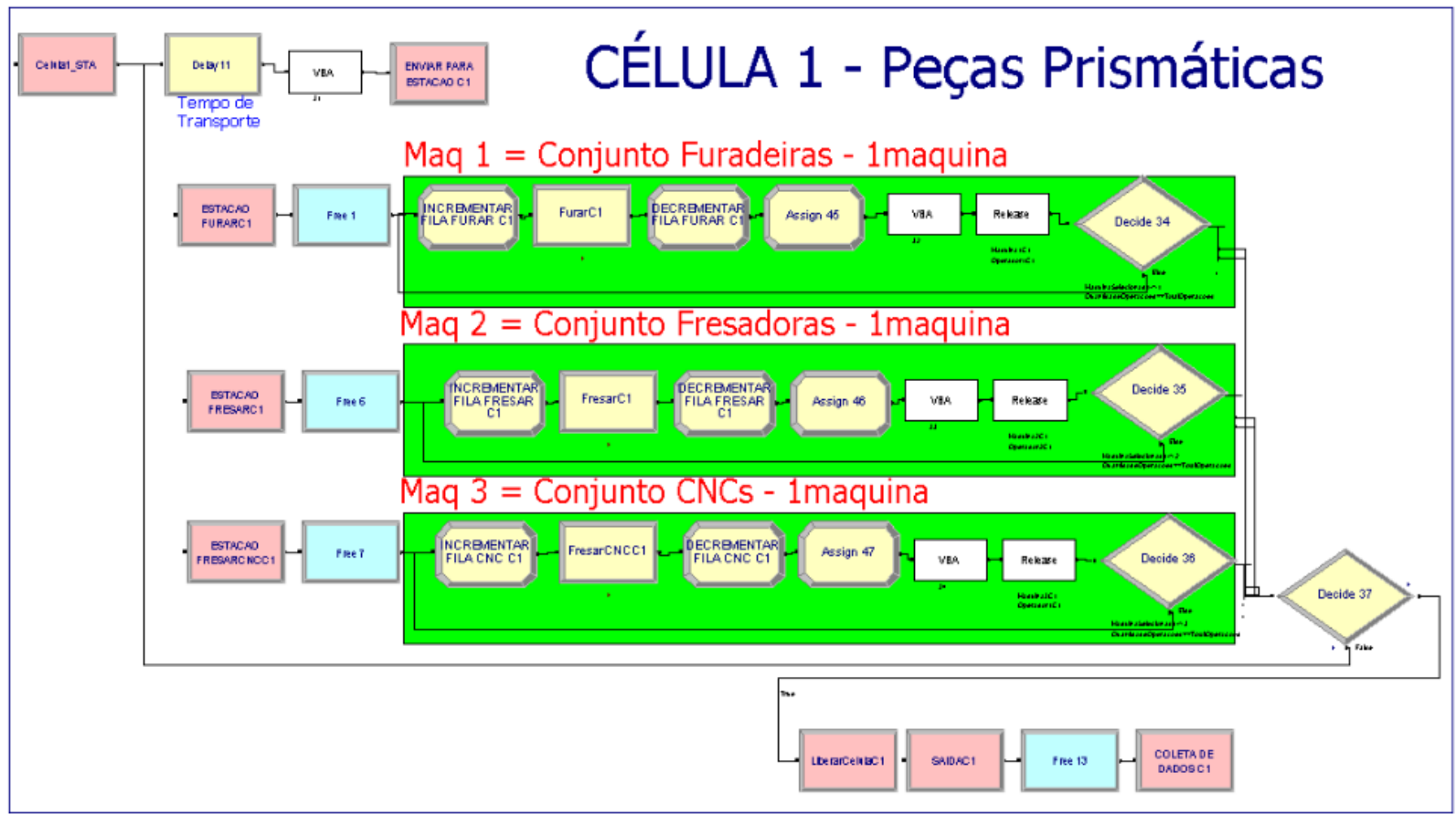

Fonte: Decker Junior (2015). 
Comparação dos projetos fatoriais completo e fracionado em um modelo de simulação de eventos discretos em um sistema de manufatura para os leiautes celular e celular virtual

Figura 13 - Célula 2 do leiaute celular tradicional elaborado no Arena

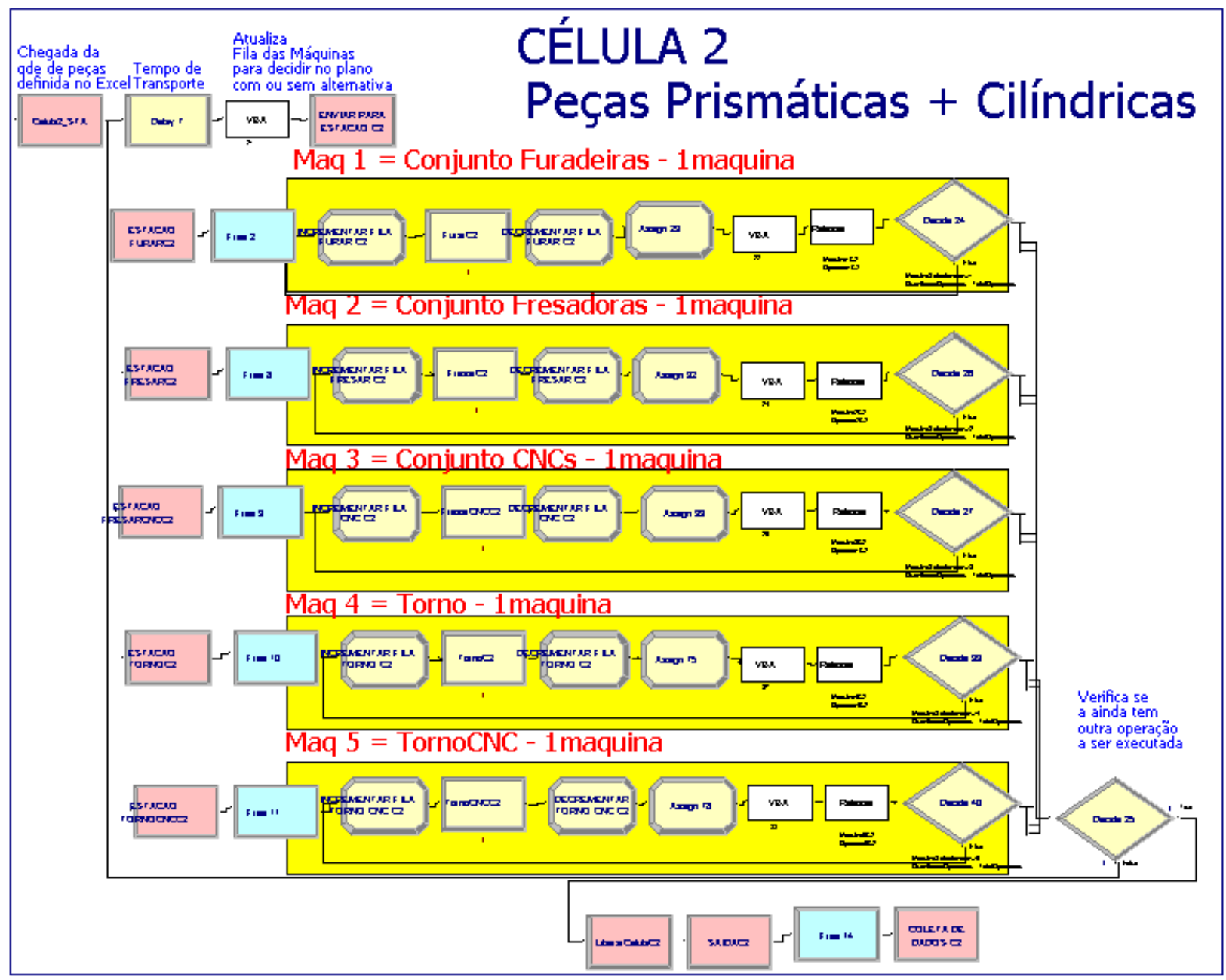

Fonte: Decker Junior (2015). 
Comparação dos projetos fatoriais completo e fracionado em um modelo de simulação de eventos discretos em um sistema de manufatura para os leiautes celular e celular virtual

Figura 14 - Célula 3 do leiaute celular tradicional elaborado no Arena

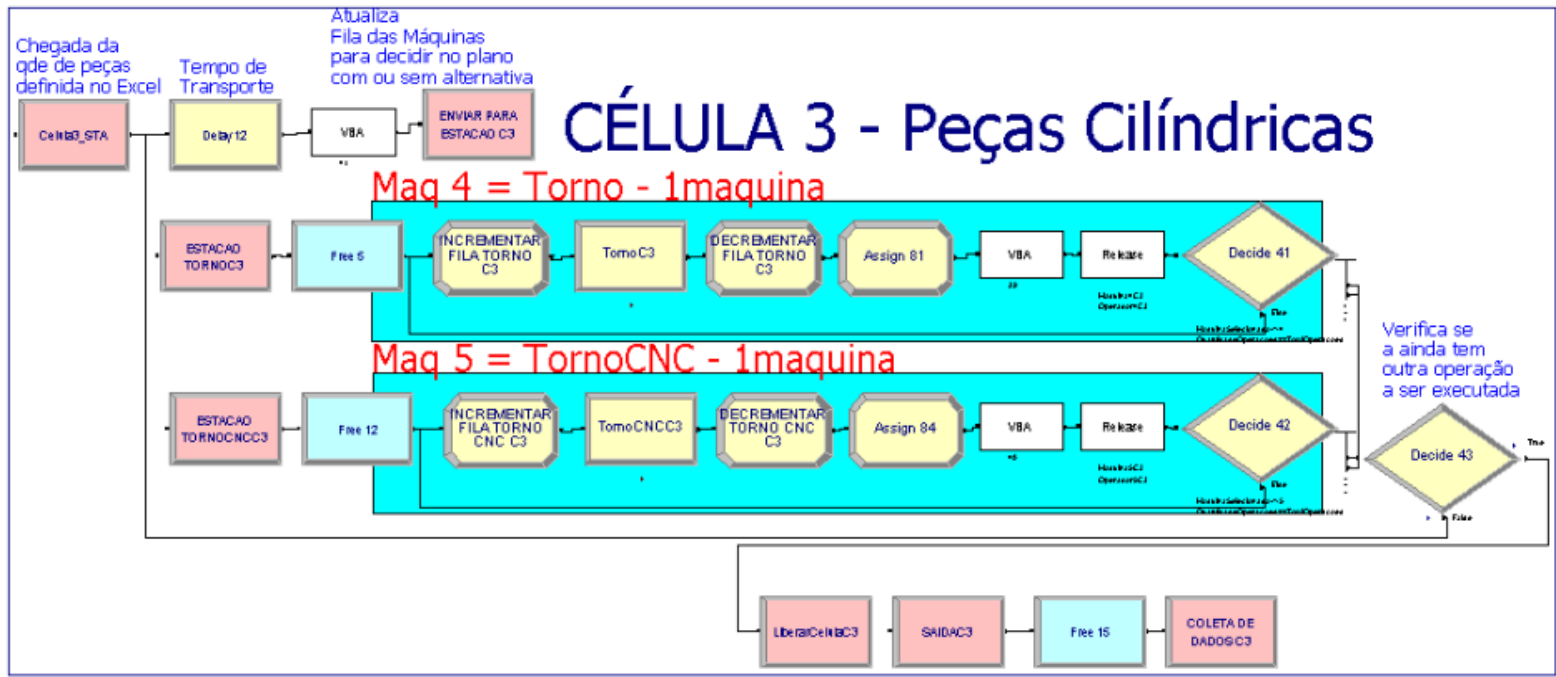

Fonte: Decker Junior (2015).

Após todas as operações realizadas, os lotes são levados até a saída do sistema pelo movimentador. É importante mencionar que o movimentador é dedicado à célula, e cada célula possui seu movimentador. A Figura 15 ilustra a fase final do modelo celular.

Figura 15 - Fase final do leiaute celular tradicional elaborado no Arena

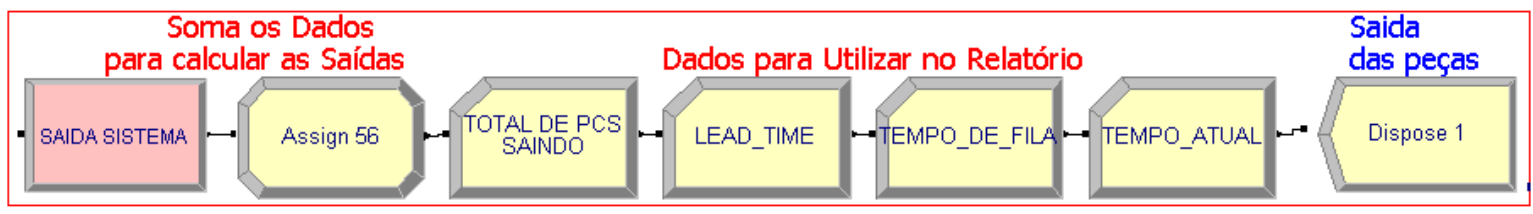

Fonte: Decker Junior (2015).

\subsection{Verificação e validação dos modelos}

Utilizaram-se as técnicas de verificação indicadas por Freitas Filho (2008). Em particular o emprego de valores constantes, de forma que os modelos passem a ser determinísticos e, assim, se possam verificar os resultados obtidos. Além disso, realizaram-se também variações sobre os dados de entrada para avaliar o desempenho do modelo com diversos parâmetros. Por último, procedeu-se uma rastreabilidade (trace) de vários procedimentos.

Um importante item verificado nos três modelos é o roteiro de fabricação e, para isso, foram feitas simulações para verificar se os modelos estavam funcionando com relação ao sequenciamento, considerando-se os lotes nos departamentos, máquinas ou células GEPROS. Gestão da Produção, Operações e Sistemas, v. 15, nº 2, p. 23 - 57, 2020. 
Comparação dos projetos fatoriais completo e fracionado em um modelo de simulação de eventos discretos em um sistema de manufatura para os leiautes celular e celular virtual

especificadas. O objetivo foi verificar se não havia nenhum erro de lógica nos modelos quanto à modelagem no Arena. Para isso, utilizou-se a técnica trace. Verificou-se se as entidades percorriam a sequência correta de máquinas e operações, sequências essas que foram previstas no padrão adotado e definidos na metodologia. O anexo A apresenta uma parte do rastreamento (trace) de uma simulação do modelo leiaute celular virtual.

Freitas Filho (2008) enuncia que a validação consiste em assegurar que o modelo e os inúmeros pressupostos e simplificações adotados no desenvolvimento do modelo, se implementados de forma correta, produzam resultados semelhantes ao observado no sistema real. Ainda segundo Freitas Filho (2008), vale ressaltar que são poucas as oportunidades em que se constrói um modelo que atue de forma idêntica ao sistema real. Na maioria das vezes, o modelo visa a análise de condições diferentes às que o sistema real está sujeito, ou, ainda, o modelo representa um sistema que não existe na realidade. Na maioria das vezes, o que se busca com o modelo é verificar o desempenho de inúmeras e diversificadas alternativas para um novo projeto. Mesmo que exista um sistema real, são poucas as possibilidades para a realização de comparações detalhadas.

Portanto, a questão da validação de modelos é importante, mas deve-se ter sempre em mente suas limitações. O importante é que se faça um delineamento das principais situações sob as quais se deseja avaliar o comportamento do modelo. O objetivo principal é aumentar a confiança nos resultados apresentados pelo modelo (FREITAS FILHO, 2008).

Quando a simulação termina, com todos os lotes simulados, armazena-se o relatório gerado pelo software Arena ${ }^{\circledR}$ com dados a serem usados para calcular a utilização de recursos. A Figura 16 ilustra um fluxograma que descreve a simulação dos modelos. 
Comparação dos projetos fatoriais completo e fracionado em um modelo de simulação de eventos discretos em um sistema de manufatura para os leiautes celular e celular virtual

Figura 16 - Fluxograma geral para a simulação dos modelos

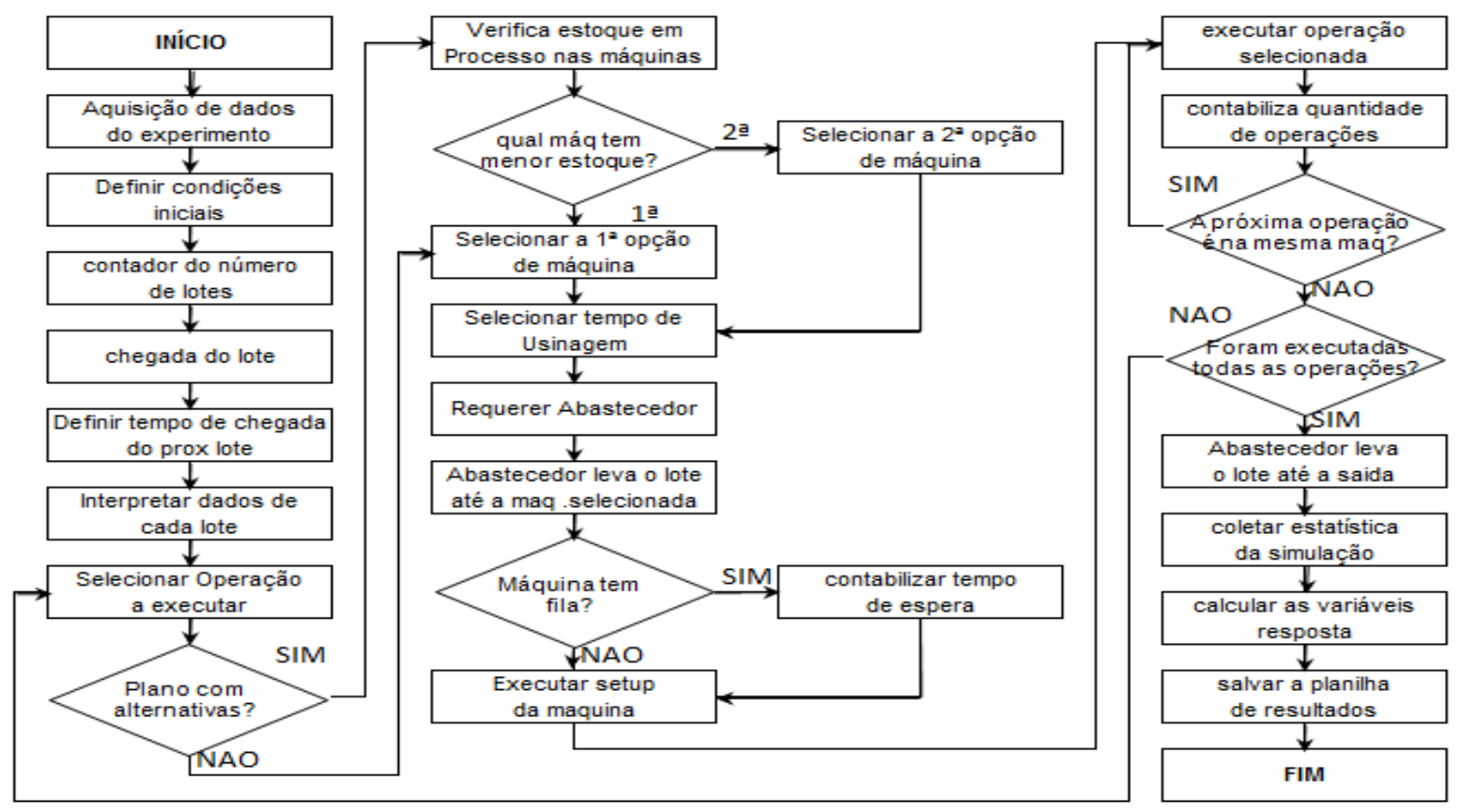

Fonte: Decker Junior et al. (2015).

Após a simulação executa-se uma análise exploratória dos dados coletados. Depois realiza-se a análise estatística por meio da Análise da Variância (ANOVA). Faz-se também a representação gráfica dos efeitos principais dos fatores de controle para a variável-resposta utilização de recursos.

Então são analisados os resultados obtidos por meio de um projeto fatorial fracionado do projeto de experimentos, e é verificada a congruência dos dados encontrados, com a aplicação de um teste t para duas amostras independentes. Objetivou-se verificar se o projeto fatorial fracionado gera os mesmos resultados que o projeto fatorial completo dos valores obtidos por Decker Junior et al. (2015).

A análise estatística foi realizada com o software R (R CORE TEAM, 2016), com auxílio do pacote FrF2 (GROEMPING, 2012) e o nível de significância adotado foi $\alpha=5 \%$. Todas as suposições para a aplicação da ANOVA e teste $\mathrm{t}$ foram verificadas, mas não são mostradas no presente trabalho. 
Comparação dos projetos fatoriais completo e fracionado em um modelo de simulação de eventos discretos em um sistema de manufatura para os leiautes celular e celular virtual

\section{RESULTADOS E DISCUSSÕES}

Num primeiro momento é necessário se fazer uma análise exploratória dos dados e verificar o comportamento dos resultados da simulação para o projeto de experimentos com relação a variável-resposta utilização de recursos (\%). Na Tabela 4 estão os resultados da análise exploratória.

Tabela 4 - Análise exploratória do percentual da utilização de recursos

\begin{tabular}{ccccc}
\hline & \multicolumn{2}{c}{ Projeto Fatorial Completo } & \multicolumn{2}{c}{ Projeto Fatorial Fracionado } \\
\cline { 2 - 5 } & $\begin{array}{c}\text { Leiaute Celular } \\
\text { Virtual }\end{array}$ & $\begin{array}{c}\text { Leiaute Celular } \\
\text { Tradicional }\end{array}$ & $\begin{array}{c}\text { Leiaute Celular } \\
\text { Virtual }\end{array}$ & $\begin{array}{c}\text { Leiaute Celular } \\
\text { Tradicional }\end{array}$ \\
\hline Valor mínimo & 0.02862 & 0.02537 & 0.02874 & 0.02564 \\
Mediana & 0.33380 & 0.27200 & 0.33150 & 0.27730 \\
Média & 0.33090 & 0.26200 & 0.33040 & 0.26080 \\
Desvio padrão & 0.124254 & 0.08964361 & 0.1237155 & 0.08916111 \\
Valor máximo & 0.55590 & 0.49970 & 0.55590 & 0.49680 \\
\hline
\end{tabular}

Fonte: Os autores.

Num segundo momento, elaborou-se a ANOVA para os resultados do projeto fatorial completo e verificaram-se quais dos oito fatores de controle (Tabela 1) do projeto de experimentos eram significativos. As Tabelas 5 e 6 apresentam o resultado da análise da variância (ANOVA) para o projeto fatorial completo dos dois tipos de leiaute.

Tabela 5 - ANOVA para o leiaute celular virtual do projeto fatorial completo

\begin{tabular}{cccccc}
\hline Parâmetros & DF & Sum Sq & Mean Sq & F-value & P-value \\
\hline F1 - Features por peça & 1 & 0.1336 & 0.1336 & 31.089 & $6.45 \mathrm{e}-08$ \\
F2 - Features duplicadas & 1 & 0.0648 & 0.0648 & 15.047 & 0.000133 \\
F3 - Tamanho do lote & 1 & 0.2591 & 0.2591 & 60.307 & $2.16 \mathrm{e}-13$ \\
F4 - Tipo de demanda & 1 & 0.0010 & 0.0010 & 0.232 & $\underline{\mathbf{0 . 6 3 0 1 9 2}}$ \\
F5 - Tipo de plano & 1 & 0.1309 & 0.1309 & 30.475 & $8.54 \mathrm{e}-08$ \\
F6 - Lotes simulados & 1 & 0.3371 & 0.3371 & 78.456 & $<2 \mathrm{e}-16$ \\
F7 - Tempo de chegada & 1 & 0.7901 & 0.7901 & 183.894 & $<2 \mathrm{e}-16$ \\
F8 - Tempo de manutenção & 1 & 1.1591 & 1.1591 & 269.766 & $<2 \mathrm{e}-16$ \\
\hline Resíduos & $\mathbf{2 4 7}$ & $\mathbf{1 . 0 6 1 3}$ & $\mathbf{0 . 0 0 4 3}$ & & \\
\hline
\end{tabular}

Fonte: Os autores. 
Comparação dos projetos fatoriais completo e fracionado em um modelo de simulação de eventos discretos em um sistema de manufatura para os leiautes celular e celular virtual

Tabela 6 - ANOVA para o leiaute celular tradicional do projeto fatorial completo

\begin{tabular}{cccccc}
\hline Parâmetros & DF & Sum Sq & Mean Sq & F-value & P-value \\
\hline F1 - Features por peça & 1 & 0.0021 & 0.0021 & 0.567 & $\underline{\mathbf{0 . 4 5 2 3 3}}$ \\
F2 - Features duplicadas & 1 & 0.0323 & 0.0323 & 8.655 & 0.00357 \\
F3 - Tamanho do lote & 1 & 0.1223 & 0.1223 & 32.739 & $3.04 \mathrm{e}-08$ \\
F4 - Tipo de demanda & 1 & 0.0004 & 0.0004 & 0.117 & $\underline{\mathbf{0 . 7 3 2 5 5}}$ \\
F5 - Tipo de plano & 1 & 0.0749 & 0.0749 & 20.062 & $1.15 \mathrm{e}-05$ \\
F6 - Lotes simulados & 1 & 0.1890 & 0.1890 & 50.596 & $1.22 \mathrm{e}-11$ \\
F7 - Tempo de chegada & 1 & 0.0885 & 0.0885 & 23.687 & $2.02 \mathrm{e}-06$ \\
F8 - Tempo de manutenção & 1 & 0.6172 & 0.6172 & 165.274 & $<2 \mathrm{e}-16$ \\
\hline Resíduos & $\mathbf{2 4 7}$ & $\mathbf{0 . 9 2 2 5}$ & $\mathbf{0 . 0 0 3 7}$ & & \\
\hline
\end{tabular}

Fonte: Os autores.

Verifica-se nas Tabelas 5 e 6 que somente os fatores F1 e F4 não são significativos. O fator F1 não é estatisticamente significativo para a utilização dos recursos, seja para o leiaute celular virtual, seja para o leiaute celular tradicional O fator de controle F1 - Quantidade de features por peça também não se mostrou estatisticamente significativo para o leiaute celular tradicional.

Nesta etapa da pesquisa, estabeleceu-se um projeto fatorial fracionado e as amostras foram coletadas da base de dados do planejamento fatorial completo. Os resultados da ANOVA estão nas Tabelas 7 e 8.

Tabela 7 - ANOVA para o leiaute celular virtual do projeto fatorial fracionado

\begin{tabular}{cccccc}
\hline Parâmetros & DF & Sum Sq & Mean Sq & F-value & P-value \\
\hline F1 - Features por peça & 1 & 0.0734 & 0.0734 & 16.779 & $7.70 \mathrm{e}-05$ \\
F2 - Features duplicadas & 1 & 0.0343 & 0.0343 & 7.836 & 0.005976 \\
F3 - Tamanho do lote & 1 & 0.1344 & 0.1344 & 30.727 & $1.82 \mathrm{e}-07$ \\
F4 - Tipo de demanda & 1 & 0.0006 & 0.0006 & 0.136 & $\underline{\mathbf{0 . 7 1 3 0 9 3}}$ \\
F5 - Tipo de plano & 1 & 0.0602 & 0.0602 & 13.759 & 0.000317 \\
F6 - Lotes simulados & 1 & 0.1708 & 0.1708 & 39.052 & $6.65 \mathrm{e}-09$ \\
F7 - Tempo de chegada & 1 & 0.3951 & 0.3951 & 90.307 & $2.82 \mathrm{e}-16$ \\
F8 - Tempo de manutenção & 1 & 0.5544 & 0.5544 & 126.719 & $<2 \mathrm{e}-16$ \\
\hline Resíduos & $\mathbf{1 1 9}$ & $\mathbf{0 . 5 2 0 6}$ & $\mathbf{0 . 0 0 4 4}$ & &
\end{tabular}

Fonte: Os autores. 
Comparação dos projetos fatoriais completo e fracionado em um modelo de simulação de eventos discretos em um sistema de manufatura para os leiautes celular e celular virtual

Tabela 8 - ANOVA para o leiaute celular tradicional do projeto fatorial fracionado

\begin{tabular}{cccccc}
\hline Parâmetros & DF & Sum Sq & Mean Sq & F-value & P-value \\
\hline F1 - Features por peça & 1 & 0.0006 & 0.00062 & 0.167 & $\underline{\mathbf{0 . 6 8 3 4 7}}$ \\
F2 - Features duplicadas & 1 & 0.0152 & 0.01516 & 4.101 & 0.04508 \\
F3 - Tamanho do lote & 1 & 0.0700 & 0.06999 & 18.933 & $2.88 \mathrm{e}-05$ \\
F4 - Tipo de demanda & 1 & 0.0003 & 0.00031 & 0.085 & $\underline{\mathbf{0 . 7 7 1 0 6}}$ \\
F5 - Tipo de plano & 1 & 0.0331 & 0.03312 & 8.959 & 0.00336 \\
F6 - Lotes simulados & 1 & 0.0984 & 0.09841 & 26.621 & $1.00 \mathrm{e}-06$ \\
F7 - Tempo de chegada & 1 & 0.0405 & 0.04053 & 10.965 & 0.00123 \\
F8 - Tempo de manutenção & 1 & 0.3116 & 0.31155 & 84.278 & $1.63 \mathrm{e}-15$ \\
\hline Resíduos & $\mathbf{1 1 9}$ & $\mathbf{0 . 4 3 9 9}$ & $\mathbf{0 . 0 0 3 7 0}$ & & \\
\hline
\end{tabular}

Fonte: Os autores.

Observa-se que, novamente, o fator F4 não é significativo, sendo possível afirmar que o fator de controle F4 - Tipo de demanda não tem influência significativa para a utilização dos recursos, tanto para o leiaute celular tradicional quanto para o leiaute celular virtual no projeto fatorial fracionado. O fator de controle F1 - Quantidade de features por peça também não foi estatisticamente significativo para o leiaute celular tradicional no projeto fatorial fracionado, mas o foi no completo.

Dentro do contexto do modelo, o fator de controle F4 - Tipo de demanda não ser significativo para a utilização de recursos pode ser explicado a partir dos níveis estabelecidos. Portanto, independentemente de se a demanda é controlada ou aleatória, os recursos serão utilizados da mesma forma e não há um impacto. Porém uma análise dos intervalos para as médias pode mostrar dentro de qual tipo de leiaute os recursos foram melhor utilizados. Para expandir essa análise poder-se-ia acrescentar mais um nível a este fator.

No leiaute celular virtual, a quantidade de features por peça (F1) é significativa para a utilização dos recursos. Quanto mais features em cada peça, maior será a utilização dos recursos, mais operações serão executadas. Isso valoriza o conceito de célula virtual já que do ponto de vista lógico a célula virtual mantêm os recursos alocados para a execução do lote de peças até que este esteja completo.

Outra análise importante a ser feita é com relação aos gráficos de efeitos principais causados pelos fatores significativos. As Figuras 17 e 18 apresentam os efeitos principais causados pelos fatores significativos tanto para o leiaute celular virtual quanto para o leiaute celular tradicional. 
Comparação dos projetos fatoriais completo e fracionado em um modelo de simulação de eventos discretos em um sistema de manufatura para os leiautes celular e celular virtual

Figura 17 - Efeitos principais para utilização de recursos leiaute celular virtual - projeto fatorial completo

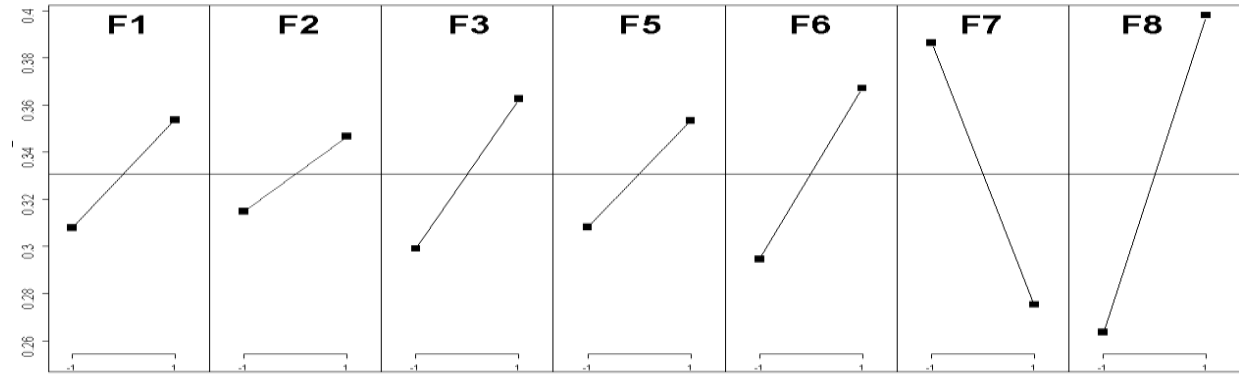

Fonte: Os autores.

Figura 18 - Efeitos principais para utilização de recursos leiaute celular tradicional - projeto fatorial completo

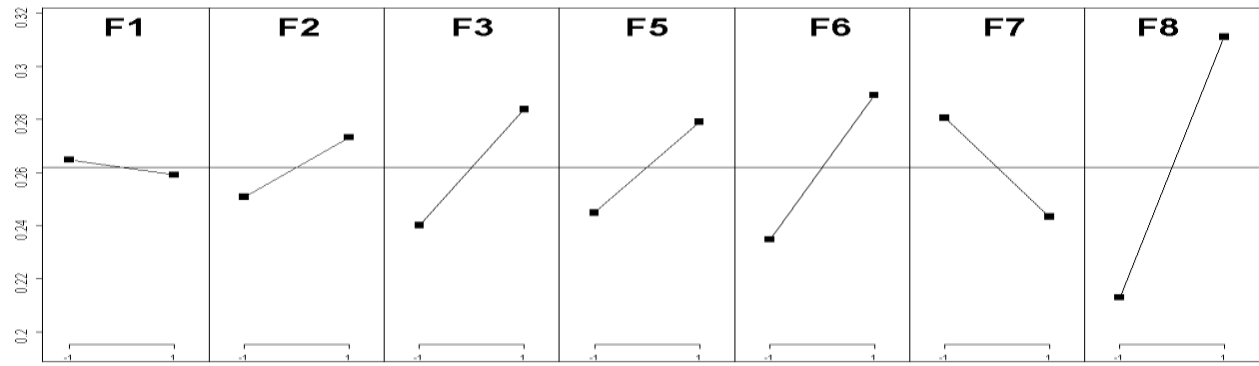

Fonte: Os autores.

As Figuras 19 e 20 apresentam os efeitos principais para o projeto fatorial fracionado do leiaute celular virtual e celular tradicional, respectivamente.

Figura 19 - Efeitos principais para utilização de recursos leiaute celular virtual - projeto fatorial fracionado

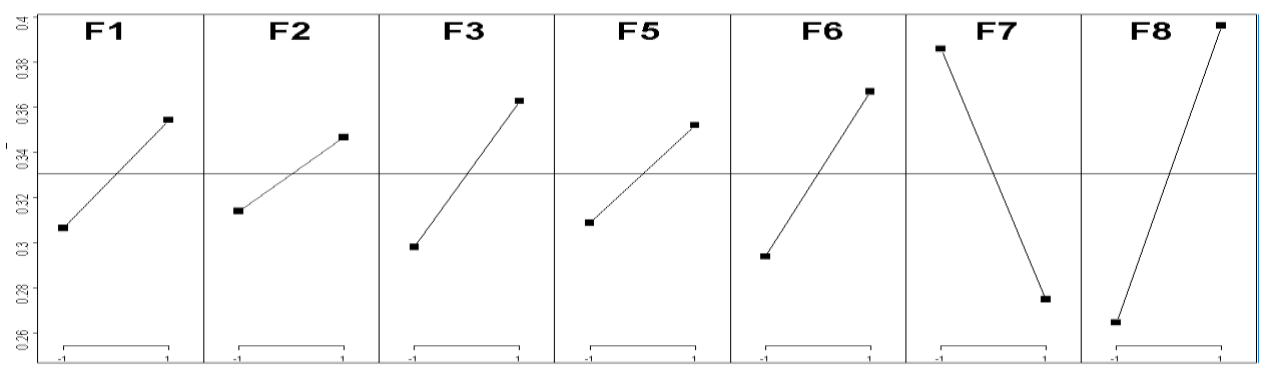

Fonte: Os autores. 
Comparação dos projetos fatoriais completo e fracionado em um modelo de simulação de eventos discretos em um sistema de manufatura para os leiautes celular e celular virtual

Figura 20 - Efeitos principais para utilização de recursos leiaute celular tradicional - projeto fatorial fracionado

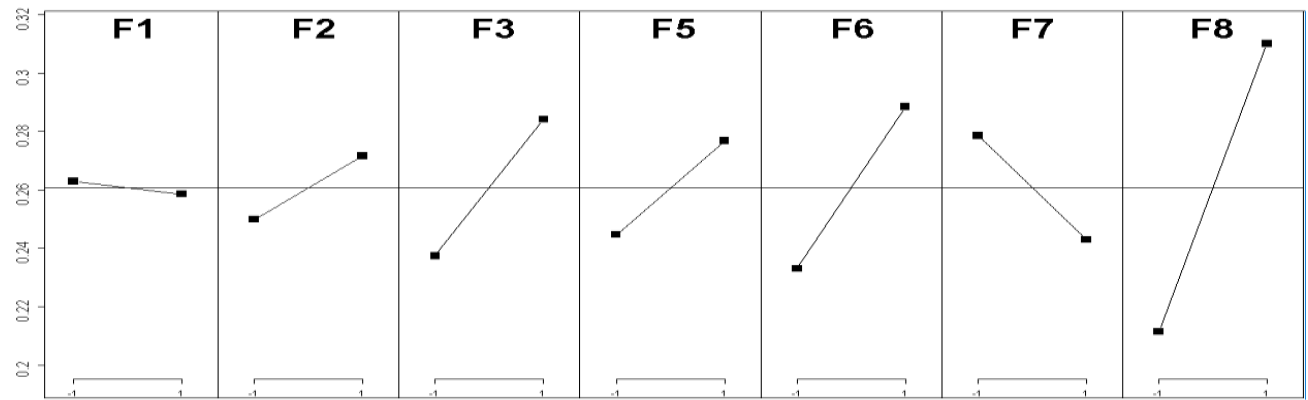

Fonte: Os autores.

Verifica-se que, de modo geral, o modelo com manutenção (F8), tempo de chegada (F7) mais curto, mais lotes (F6) e com mais peças (F3), plano com alternativas (F5) com mais features duplicadas $(\mathrm{F} 2)$ resulta no aumento da utilização dos recursos.

Além da análise para verificar se o projeto fatorial fracionado estima os mesmos resultados significativos do projeto fatorial completo, a comparação dos leiautes se torna essencial sob o ponto de vista dos sistemas de manufatura.

A comparação entre os leiautes serve para verificar se existe uma diferença significativa entre a utilização de recursos e isso foi feito a partir do Teste t de Student, para amostras independentes. A partir dos resultados, pode-se afirmar que o leiaute celular virtual gera uma maior utilização de recursos (p-valor $<0,05)$ para os dois projetos fatoriais. Os intervalos de $95 \%$ de confiança para a média da utilização de recursos complementam a análise.

O intervalo para a utilização de recursos no leiaute celular tradicional é de $(25,0 \% \leq \mu$ $\leq 27,3 \%)$, enquanto que no leiaute celular virtual o intervalo é de $(31,5 \% \leq \mu \leq 34,6 \%)$.

Para os dados do projeto fatorial fracionado, os intervalos para a utilização de recursos do leiaute celular tradicional é de $(24,5 \% \leq \mu \leq 27,6 \%)$ e para o leiaute celular virtual é de $(30,8 \% \leq \mu \leq 35,2 \%)$.

Observa-se, por meio dos intervalos de confiança, que o leiaute celular virtual apresenta valores médios superiores de utilização de recursos em relação ao leiaute celular 
Comparação dos projetos fatoriais completo e fracionado em um modelo de simulação de eventos discretos em um sistema de manufatura para os leiautes celular e celular virtual

tradicional. Em média, a utilização de recursos no leiaute celular virtual foi $20 \%$ superior à do celular tradicional.

Reaes (2008) cita que na comparação entre os leiautes celular virtual e tradicional a ocupação dos operadores foi maior no leiaute celular tradicional, porém nesta pesquisa consideraram-se, além dos operadores, todos os recursos do leiaute, as máquinas e os movimentadores, resultando num resultado global superior do leiaute virtual. O melhor desempenho do celular virtual nesse estudo concorda com Hamedi et al. (2012a, 2012c).

Os resultados da análise de sensibilidade realizada (Figura 21) indicam que as duas maiores contribuições na utilização de recursos para o leiaute celular são o tempo de manutenção (F8) e o tempo de chegada (F7). Para o leiaute celular tradicional, os resultados se encontram na Figura 22 e os dois fatores de maior importância são o tempo de manutenção (F8) e a quantidade de lotes simulados (F6).

Figura 21 - Comparação dos resultados da análise de sensibilidade para o leiaute celular virtual - projeto fatorial completo e fracionado

\begin{tabular}{|c|c|}
\hline F8 - Tempo de manutenção dos equipamentos & 126.719 \\
\hline F7 - Tempo para a chegada do próximo lote & 90.307 \\
\hline F6 - Quantidade total de lotes simulados em cada replicação & 39.05278 .456 \\
\hline F3 - Quantidade de peças contidas em cada lote & 30.72760 .307 \\
\hline F1 - Quantidade de features por peça & ECompleto $\quad$ Fracionado \\
\hline F5 - Plano de processos utilizado na simulação com ou sem alternativas & $13.759^{30.475}$ \\
\hline F2 - Quantidade de duplicações de uma mesma feature em cada peça & $7.836^{1074}$ \\
\hline
\end{tabular}

Fonte: Os autores. 
Comparação dos projetos fatoriais completo e fracionado em um modelo de simulação de eventos discretos em um sistema de manufatura para os leiautes celular e celular virtual

Figura 22 - Comparação dos resultados da análise de sensibilidade para o leiaute celular tradicional - projeto fatorial completo e fracionado

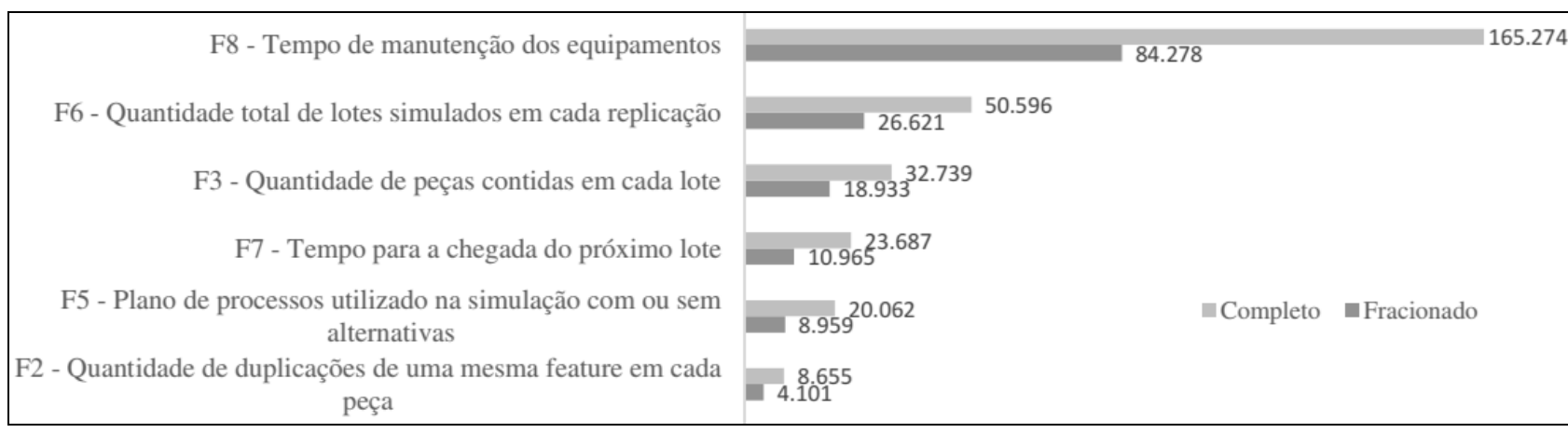

Fonte: Os autores.

Pode ser verificado que os resultados de classificação para os índices de sensibilidade foram os mesmos para o projeto fatorial completo e fracionado. Concordam também com a análise dos efeitos principais, o que é esperado, mas a AS permite uma quantificação dessa priorização. Chama a atenção a importância do fator F8 - Tempo de manutenção. Isso se deve ao fato de que, no modelo, os níveis deste fator significam que os equipamentos utilizados possuem ou não tempo de manutenção.

Caso a empresa utilize um plano de manutenção adequado, os equipamentos se tornam $100 \%$ disponíveis, o que reflete numa maior disponibilidade do tempo das máquinas, em consequência, na utilização dos recursos. Do contrário, caso a empresa não utilize um plano de manutenção temos que considerar intervenções corretivas, o que ocasiona parada de máquina. Essas paradas deixam os recursos ociosos enquanto algum sobressalente é trocado, impactando também na utilização de recursos.

O fator F7 - Tempo de chegada, como já mencionado, se refere ao tempo de chegada entre cada lote a ser fabricado. Faz sentido ele ser um fator com alto nível de importância, pois dependendo do tempo que a fabricação de cada lote de peças demora para iniciar-se, pode haver ociosidade dos recursos. Os resultados reforçam a importância da manutenção para os dois leiautes. Priorizar recursos para a manutenção preventiva irá resultar na melhor utilização dos recursos, ou seja, mais benefícios para a empresa.

Da mesma forma, da Figura 13 pode-se concluir alguns pontos importantes sobre os índices de sensibilidade encontrados para o leiaute celular tradicional. Neste caso, o fator F8 Tempo de manutenção também foi o índice de sensibilidade com maior valor. Porém o GEPROS. Gestão da Produção, Operações e Sistemas, v. 15, nº 2, p. 23 - 57, 2020. 
Comparação dos projetos fatoriais completo e fracionado em um modelo de simulação de eventos discretos em um sistema de manufatura para os leiautes celular e celular virtual

segundo fator com maior índice de sensibilidade foi F6 - Lotes simulados. Esse fator se refere à quantidade de lotes que são simulados de cada vez em cada modelo: no nível baixo são simulados 50 lotes, enquanto no nível alto são 200 lotes. Essa variação na quantidade de lotes simulados influencia a utilização de recursos principalmente no leiaute celular tradicional, pois o arranjo dos equipamentos neste leiaute faz com que cada lote acesse a célula e seja confeccionado. Quanto maior a quantidade de lotes distribuídos nas células maior a ocupação dos recursos. Os resultados após a análise de sensibilidade mostram que o projeto fatorial completo e o projeto fatorial fracionado obtiveram, de modo global, resultados semelhantes, corroborando o objetivo deste estudo.

Ainda, os resultados obtidos concordam com a literatura, com relação a adequabilidade do método proposto. De acordo com os autores, os métodos de DOE, podem não oferecer uma solução adequada se aplicados isoladamente, produzindo melhores resultados em conjunto com outras técnicas de otimização (SOUZA JR et al., 2019).

Sumarizando os resultados, pode-se concluir que a simulação mostrou que o leiaute celular tem maior utilização de recursos. Além disso, um projeto fracionado levou aos mesmos resultados que um fatorial completo para o leiaute celular virtual. A Análise de Sensibilidade permitiu encontrar, dentre os diversos fatores significativos, aqueles que mais contribuem para a utilização dos recursos.

Além da contribuição dessa pesquisa para aplicações práticas, a metodologia aqui proposta (Planejamento de experimentos, Simulação e Análise de sensibilidade) é uma alternativa a ser avaliada no ensino de Engenharia, particularmente em metodologias ativas de aprendizagem.

É possível integrar num único projeto vários conteúdos importantes para os acadêmicos de Engenharia Mecânica e de Engenharia de Produção. A organização do leiaute produtivo, o desenho de experimentos, a utilização de projetos fracionados e a priorização de fatores podem auxiliar os alunos a compreender a importância da interdisciplinaridade e do planejamento para o processo produtivo.

\section{CONCLUSÕES}

Neste trabalho foram utilizados os métodos de projetos de experimentos, com projeto fatorial completo e projeto fatorial fracionado, através de dados da simulação a eventos discretos de um sistema de manufatura configurado através de dois diferentes leiautes. Foram 
Comparação dos projetos fatoriais completo e fracionado em um modelo de simulação de eventos discretos em um sistema de manufatura para os leiautes celular e celular virtual

modelados os leiautes celular tradicional e celular virtual e foram avaliados e comparados os resultados e o comportamento da utilização dos recursos para cada um dos modelos.

Os resultados da pesquisa reforçam a importância da simulação de sistemas de manufatura. Este artigo contribui para mostrar a superioridade da simulação leiaute celular na utilização de recursos. Além disso, mostra a aplicabilidade de um planejamento experimental reduzido, que levou aos mesmos resultados que um planejamento completo. Por fim, a Análise de Sensibilidade permitiu encontrar, dentre os diversos fatores significativos, aqueles que mais contribuem para a utilização dos recursos.

Os resultados mostram que o projeto fatorial completo e o projeto fatorial fracionado obtiveram os mesmos resultados para o leiaute celular virtual. Foi possível mostrar que dos oito fatores de controle inicialmente estabelecidos, o fator F4 - Tipo de demanda não foi significativo para ambos os leiautes, tanto no projeto fatorial completo quanto no projeto fatorial fracionado. Considerando os fatores significativos, o modelo com manutenção (F8), tempo de chegada (F7) mais curto, mais lotes (F6) e com mais peças (F3), plano com alternativas (F5) com mais features duplicadas (F2) resulta no aumento da utilização dos recursos. O tempo de manutenção e o de chegada das peças foram os fatores com maior importância para todos os modelos, de acordo com a análise de sensibilidade. A manutenção é um aspecto importante nos processos e deve ser investigada de modo a otimizar os resultados esperados por uma organização.

O leiaute celular virtual teve vantagem sobre o leiaute celular tradicional. Vale ressaltar que a montagem do modelo não promoveu o fluxo contínuo, pois a fabricação acontecia por lotes de peças. Isso pode ser visto como ponto negativo para o leiaute celular tradicional. Por fim, nota-se que o leiaute celular virtual pode ter vantagens em alguns cenários pouco explorados nas indústrias. Em tempos de indústria 4.0 novos cenários devem aparecer e é importante que se tenham alternativas para analisar sua viabilidade e performance.

Assim, explorar diferentes cenários em processos de manufatura, aplicando simulação e planejamento de experimentos se configura numa opção para otimizar a produção, minimizando as perdas de tempo e recursos. Para a continuidade dos trabalhos pode ser analisada a utilização de outros planejamentos, como os ortogonais.

Além da contribuição dessa pesquisa para aplicações práticas, a metodologia aqui proposta (Planejamento de experimentos, Simulação e Análise de sensibilidade) é uma GEPROS. Gestão da Produção, Operações e Sistemas, v. 15, nº 2, p. 23 - 57, 2020. 
Comparação dos projetos fatoriais completo e fracionado em um modelo de simulação de eventos discretos em um sistema de manufatura para os leiautes celular e celular virtual

alternativa a ser avaliada no Ensino de Engenharia, particularmente em metodologias ativas de aprendizagem.

É possível integrar num único projeto vários conteúdos importantes para os acadêmicos de Engenharia Mecânica e de Engenharia de Produção. A organização do leiaute produtivo, o desenho de experimentos, a utilização de projetos fracionados e a priorização de fatores podem auxiliar os alunos a compreender a importância da interdisciplinaridade e do planejamento para o processo produtivo.

\section{Referências}

AL-AOMAR, R.; AL-OKAILY, A. A GA-based parameter design for single machine turning process with high-volume production. Computers and Industrial Engineering, v.50, p.317337, 2006.

ANTONY, J. Design of Experiments for Engineers and Scientists. Elsevier, 2014.

ARKAT, J.; GHAHVE, H. Scheduling of virtual manufacturing cells with outsourcing allowed. International Journal of Computer Integrated Manufacturing, v.27, n.12, p.1079-1089, 2014.

COSTA, R. F. da S. Abordagem sistemática para avaliação econômica de cenários para modelos de simulação discreta em manufatura. Dissertação. 2010. 105f. (Mestrado em Engenharia de Produção e Gestão) - Instituto de Engenharia de Produção e Gestão, Universidade Federal de Itajubá, Itajubá, MG, Brasil, 2010.

DECKER JUNIOR, C. Comparação dos leiautes funcional, celular e celular virtual utilizando plano de processos com alternativas por meio de simulação e projeto de experimentos. Dissertação. 2015. 120f. (Mestrado em Engenharia Mecânica) - Departamento de Engenharia Mecânica, Universidade Federal de Santa Catarina, Florianópolis -SC, Brasil, 2015.

DECKER JUNIOR, C.; FERREIRA, J. C. E.; SILVA, G. J.; LOUREIRO, F. M. Comparison of Traditional and Virtual Cell Layouts Using Alternative Process Plans Through Simulation and Design of Experiments. In: ABCM INTERNATIONAL CONGRESS OF MECHANICAL ENGINEERING. Anais...23 ${ }^{\text {rd }}, 2015$, Rio de Janeiro.

DEVISE, O.; PIERREVAL, H. Indicators for measuring performances of morphology and material handling system in flexible manufacturing systems. International Journal of Production Economics, v.64, p.209-218, 2000.

DZIAK, J. J.; NAHUM-SHANI, I.; COLLINS, L. M. (2012). Multilevel factorial experiments for developing behavioral interventions: Power, sample size, and resource considerations. Psychological Methods, v. 17, n.2, p. 153-175, 2012. 
Comparação dos projetos fatoriais completo e fracionado em um modelo de simulação de eventos discretos em um sistema de manufatura para os leiautes celular e celular virtual

EKREN, B.Y.; ORNEK, A. M. A simulation based experimental design to analyse factors affecting production flow time. Simulation Modelling Practice and Theory, v.16, p.278293, 2008.

DROLET, J.; MARCOUX, Y.; ABDULNOUR, G. Simulation-based performance comparison between dynamic cells, classical cells and job shops: a case study. International Journal of Production Research, v.46, n.2, p.509-536, 2008.

FREITAS FILHO, P. Introdução a Modelagem e Simulação de Sistemas: com aplicações em Arena®. $2^{\mathrm{a}}$ Ed. Revisada e atualizada. Florianópolis: Visual Books, 2008.

GOMES, J. H. F. Análise e otimização da soldagem de revestimento de chapas de aço carbono utilizando arame tubular inoxidável austenítico. 2010. 136 p. Dissertação (Mestrado em Engenharia de Produção). Universidade Federal de Itajubá (UNIFEI), Itajubá, MG, Brasil, 2010.

GREENE, T.J.; SADOWSLI, R.P. Cellular Manufacturing Control. Journal of Manufacturing System, v.2, n.2, p.137-145, 1982.

HAMBY, D.M. A review of techinques for parameter sensitivity analysis of environmental models, Environmental Monitoring and Assessment, v.32, p.135-134, 1994.

HAMEDI, M.; ESMAEILIAN, G. R.; ISMAIL. N.; ARIFFIN, M. K. A. Capability-based virtual cellular manufacturing systems formation in dual-resource constrained settings using tabu search. International Journal of Computers \& Industrial Engineering, v.62, p.953971, 2012a.

HAMEDI, M.; ESMAEILIAN, G. R.; ISMAIL. N.; ARIFFIN, M. K. A. A survey on formation of virtual cellular manufacturing systems (VCMSs) and related issues. Scientific Research and Essays, v.7, n.40, p.3316-3328, 2012b.

HAMEDI, M.; ESMAEILIAN, G. R.; ISMAIL. N.; ARIFFIN, M. K. A. Virtual cellular manufacturing system based on resource element approach and analyzing its performance over different basic layouts. International Journal of Industrial Engineering Computations, v.3, p.265-276, 2012c.

JAYACHITRA, R.; REVATHY, A.; PRASAD, P. S. S. A Fuzzy Programming Approach for Formation of Virtual Cells under Dynamic and Uncertain Conditions. International Journal of Engineering Science and Technology, v.2, n.6, p.1708-1724, 2010.

KHILWANI, N.; ULUTAS, B. H.; ISLIER, A. A.; TIWARI, M. K. A Methodology to Design Virtual Cellular Manufacturing Systems. Journal of Intelligent Manufacturing, v.22, n.4, p.533-544, 2011.

KLEIJNEN, J.P.C.; SANCHEZ, S.M.; LUCAS, T.W.; CIOPPA, T.M. State-of-the-Art Review: A User's Guide to the Brave New World of Designing Simulation Experiments. Journal on Computing, v.17, n.3, p.263-289, 2005. 
Comparação dos projetos fatoriais completo e fracionado em um modelo de simulação de eventos discretos em um sistema de manufatura para os leiautes celular e celular virtual

MCLEAN, C. R.; BLOOM, H.; HOPP, T. H.; The Virtual Manufacturing Cel. Proceedings of fourth IFAC/IFIP. In: CONFERENCE ON INFORMATION CONTROL PROBLEMS IN MANUFACTURING TECHNOLOGY. Anais... Gaithersburg, MD, 1982.

MONTEVECHI, J. A. B.; PINHO, A. F.; LEAL, F.; MARINS, F. A. S. Application of design of experiments on the simulation of a process in an automotive industry. In: WINTER SIMULATION CONFERENCE, PROCEEDINGS. Anais... Washington, DC, USA, 2007.

MONTGOMERY, D. C. Introdução ao Controle Estatístico da Qualidade. 4 ed. Rio de Janeiro: LTC, 2009.

NEGAHBAN, A.; SMITH, J. S. Simulation for manufacturing system design and operation: Literature review and analysis. Journal of Manufacturing Systems, v. 33, n. 2, p. 241-261, 2014.

POLENGHI, A.; FUMAGALLI, L.; RODA, I. Role of simulation in industrial engineering: focus on manufacturing systems. IFAC-Papers On Line, v. 51, n. 11, p. 496-501, 2018.

REAES, P. A. Comparação de desempenho entre as configurações celular, funcional e celular virtual através de simulação e projeto de experimentos. 180f. Tese (Doutorado em Engenharia Mecânica) - Departamento de Engenharia Mecânica, Universidade Federal de Santa Catarina, Florianópolis -SC, Brasil, 2008.

REZAZADEH, H., GHAZANFARI, M.; SADJADI, S.J.; ARYANEZHAD, M.B.; MAKUI, A. Linear Programming Embedded Particle Swarm Optimization for Solving an Extended Model of Dynamic Virtual Cellular Manufacturing Systems. Journal of Applied Research and Technology, v.7, n.1, p.83-108, 2009.

SALTELLI, A. Sensitivity Analysis for Importance Assessment, Risk Analysis, v.22, n.3, p.1-12, 2002.

SOUSA JUNIOR, W. T. et al. Discrete simulation-based optimization methods for industrial engineering problems: A systematic literature review. Computers \& Industrial Engineering, v. 128, p. 526-540, 2019.

YADAV, A.; JAYSWAL, S. C. Evaluation of batching and layout on the performance of flexible manufacturing system. The International Journal of Advanced Manufacturing Technology, v. 101, n. 5-8, p. 1435-1449, 2019.

WANG, Z.; YANG, J.; WANG, G.; ZHANG, G. Proceedings of the institution of Mechanical Engineers, Part C: Journal of Mechanical Engineering Science, 2011. 
Comparação dos projetos fatoriais completo e fracionado em um modelo de simulação de eventos discretos em um sistema de manufatura para os leiautes celular e celular virtual

\section{ANEXO A - Parte do rastreamento (trace) referente a uma simulação do modelo leiaute celular virtual}

SIMAN System Trace Beginning at Time: 0.0

Seq\# Label Block System Status Change

Time: 0 Entity: 2

$1112 \$$ CREATE

Entity Type set to Entity 1

Next creation scheduled at time 182.71618

Batch of 1 Entity 1 entities created

2 113\$ ASSIGN

Create 1 . Numberout set to 1.0

$30 \$ V B A$

VBA event number 1 called

NumeroPeca set to 1.0

Tipoplano set to 1.0

Totaloperacoes set to 9.0

Quantidadelote set to 31.0

TempoProcessamento set to 0.0

Sequência $(1,1)$ set to 4.0

Tempo $(1,1)$ set to 0.50265482

Sequência $(2,1)$ set to 5.0

Tempo $(2,1)$ set to 0.70371675

Sequência $(1,2)$ set to 2.0

Tempo $(1,2)$ set to 2.2266727

Sequência $(2,2)$ set to 3.0

Tempo $(2,2)$ set to 3.1173418

Sequência $(1,3)$ set to 4.0

Tempo $(1,3)$ set to 2.2619467

Sequência $(2,3)$ set to 5.0

Tempo $(2,3)$ set to 3.1667254

Sequência $(1,4)$ set to 3.0

Tempo $(1,4)$ set to 2.6744086

Sequência $(2,4)$ set to 2.0

Tempo $(2,4)$ set to 3.744172

Sequência $(1,5)$ set to 2.0

Tempo $(1,5)$ set to 3.9983907

Sequência $(2,5)$ set to 3.0

Tempo $(2,5)$ set to 5.5977469

Sequência $(1,6)$ set to 1.0

Tempo $(1,6)$ set to 2.2619467

Sequência $(2,6)$ set to 2.0

Tempo $(2,6)$ set to 3.1667254

Sequência $(1,7)$ set to 4.0

Tempo $(1,7)$ set to 0.92775158

Sequência $(2,7)$ set to 5.0

Tempo $(2,7)$ set to 1.2988522

Sequência $(1,8)$ set to 3.0

Tempo $(1,8)$ set to 8.2224374

Sequência $(2,8)$ set to 2.0

Tempo $(2,8)$ set to 11.511412

Sequência $(1,9)$ set to 5.0

Tempo $(1,9)$ set to 2.6329538

Sequência $(2,9)$ set to 4.0

Tempo $(2,9)$ set to 3.6861354

TempoProcessamentoTotal set to 25.709162

TempoProcessamentoParcial set to 0.0

TempoProximoLoteMin set to 159.3968

TempoProximoLoteMax set to 239.0952

RESERVAR MAQ1 set to 0.0

RESERVAR_MAQ2 set to 0.0

RESERVAR MAQ3 set to 0.0

RESERVAR_MAQ4 set to 0.0

RESERVAR MAQ5 set to 0.0

RESERVAR_MAQ4 set to 25.0

$\operatorname{SEQUENNCI\overline {A}}$ NOVA $(1,1)$ set to 4.0

TEMPO NOVO $(1,1)$ set to 0.50265482

RESERV̄AR_MAQ2 set to 25.0 
Comparação dos projetos fatoriais completo e fracionado em um modelo de simulação de eventos discretos em um sistema de manufatura para os leiautes celular e celular virtual

SEQUÊNCIA NOVA $(1,2)$ set to 2.0 TEMPO_NOVO $(1,2)$ set to 2.2266727 RESERVAR MAQ4 set to 25.0

SEQUÊNCIA NOVA $(1,3)$ set to 4.0 TEMPO_NOVO $(1,3)$ set to 2.2619467 RESERVAR MAQ3 set to 25.0

SEQUÊNCIĀ NOVA $(1,4)$ set to 3.0 TEMPO NOVO $(1,4)$ set to 2.6744086 RESERVAR MAQ2 set to 25.0

$\operatorname{SEQUENNCI\overline {A}}$ NOVA $(1,5)$ set to 2.0 TEMPO NOVO $(1,5)$ set to 3.9983907 SEQUÊN̄CIA NOVA $(1,6)$ set to 1.0 TEMPO_NOVO $(1,6)$ set to 2.2619467 RESERVAR MAQ1 set to 25.0

RESERVAR MAQ4 set to 25.0 $\operatorname{SEQUENNCI\overline {A}}$ NOVA $(1,7)$ set to 4.0 TEMPO NOVO $(1,7)$ set to 0.92775158 RESERVAR_MAQ3 set to 25.0 SEQUENNCIA $\operatorname{NOVA}(1,8)$ set to 3.0 TEMPO NOVO $(1,8)$ set to 8.2224374 RESERVAR_MAQ5 set to 25.0 SEQUENNCIA NOVA $(1,9)$ set to 5.0 TEMPO NOVO $(1,9)$ set to 2.6329538 Time: 0 Entity: 2

4 43\$ BRANCH

Selecting at most 1 of 2 branches IF: Entity 2 sent to $116 \$$

$5116 \$$ ASSIGN

MAQ 1. NumberOut True set to 1.0 Entity transferred to block $72 \$$ $772 \$$ ASSIGN

FILAMAQ1 set to 1.0

POSFILA1 set to 1.0

844 BRANCH

Selecting at most 1 of 2 branches

IF: Entity 2 sent to 118 \$

9118 \$SSIGN

MAQ 2. NumberOut True set to 1.0

Entity transferred to block 73\$

11 73\$ ASSIGN

FILAMAQ2 set to 1.0

POSFILA2 set to 1.0

12 45\$ BRANCH

Selecting at most 1 of 2 branches

IF: Entity 2 sent to 120 \$

$13120 \$$ ASSIGN

MAQ_3.NumberOut True set to 1.0

Entity transferred to block 74\$

1574 \$ ASSIGN

FILAMAQ3 set to 1.0

POSFILA3 set to 1.0

16 66\$ BRANCH

Selecting at most 1 of 2 branches

IF: Entity 2 sent to $122 \$$

17 122\$ ASSIGN

MAQ 4.NumberOut True set to 1.0

Entity transferred to block 75\$

$1975 \$$ ASSIGN

FILAMAQ4 set to 1.0

POSFILA4 set to 1.0

20 67\$ BRANCH

Selecting at most 1 of 2 branches

IF: Entity 2 sent to $124 \$$

21 124\$ ASSIGN

MAQ 5. NumberOut True set to 1.0

Entity transferred to block $76 \$$

$2376 \$$ ASSIGN

FILAMAQ5 set to 1.0

POSFILA5 set to 1.0

24 38\$ VBA

VBA event number 16 called

MaquinaSelecionada set to 4.0

GEPROS. Gestão da Produção, Operações e Sistemas, v. 15, nº 2, p. 23 - 57, 2020 
Comparação dos projetos fatoriais completo e fracionado em um modelo de simulação de eventos discretos em um sistema de manufatura para os leiautes celular e celular virtual

PROXIMA ESTACAO set to 4.0

TempoPrōcessamento set to 0.50265482

Time: 0 Entity: 2

$2537 \$$ STATION

Entity 2 entered station ENTRADA_SISTEMA.Station

$26126 \$$ DELAY

Delayed by 0.0 until time 0.0

27 132\$ DELAY

Delayed by 0.0 until time 0.0

281 1\$ DELAY

Delayed by 40.779521 until time 40.779521

Time: 40.779521 Entity: 2

29 39\$ DELAY

Delayed by 0.0 until time 40.779521

$30150 \$$ QUEUE

Entity 2 sent to next block

31 154\$ REQUEST

Tally SAIDA_ENTRADA_SISTEMA.Queue.WaitingTime recorded 0.0

MOVIMENTADO $\bar{R}$ reques $\bar{t}$ at station ENTRADA SISTEMA.Station

MOVIMENTADOR(1) to arrive at time 40.779521 at block $154 \$$

32 142\$ DELAY

Delayed by 0.0 until time 40.779521

33144 \$ TRANSPORT

MOVIMENTADOR(1) transport to station DEPT 4

MOVIMENTADOR (1) to arrive at time $42.7795 \overline{2} 1$ at block $57 \$$

Time: 42.779521 Entity: 2

134 57\$ STATION

Entity 2 entered station DEPT 4

135 437\$ DELAY

Delayed by 0.0 until time 42.779521

136 59\$ FREE

MOVIMENTADOR (1) freed

MOVIMENTADOR number available increased to 3

137 56\$ DELAY

Delayed by 3.710403 until time 46.489924

Time: 46.489924 Entity: 2

138 49\$ ASSIGN

FilaTornoCl set to 15.5823

139 90\$ BRANCH

Selecting at most 1 of 2 branches

IF: Branch not selected

ELSE: Entity 2 sent to 439 \$

141 439\$ ASSIGN

Decide 44.Numberout False set to 1.0

Entity transferred to block $84 \$$

158 84\$ QUEUE

Entity 2 sent to next block

159 100\$ SEIZE

Tally FILAMAQUINA4.WaitingTime recorded 0.0

Seized 1.0 unit(s) of resource Maquina4C1

Seized 1.0 unit(s) of resource Operador4C1

$160110 \$$ VBA

VBA event number 28 called

Time: 46.489924 Entity: 2

$14346 \$$ ASSIGN

TornoC1.NumberIn set to 1.0

TornoC1.WIP set to 1.0

144 441\$ DELAY

Delayed by 30.694522 until time 77.184446

Time: 77.184446 Entity: 2

145 440\$ RELEASE

Maquina $4 \mathrm{Cl}$ available increased by 1.0 to 2.0

Operador $4 \mathrm{Cl}$ available increased by 1.0 to 2.0

146 488\$ ASSIGN

Tornoc1. NumberOut set to 1.0

TornoC1.WIP set to 0.0

$14750 \$$ ASSIGN

FilaTornoc1 set to 0.0

$148 \quad 47 \$$ ASSIGN

QuantidadeOperacoes set to 1.0

GEPROS. Gestão da Produção, Operações e Sistemas, v. 15, nº 2, p. 23 - 57, 2020 